\title{
Capacity Bounds and High-SNR Capacity of MIMO Intensity-Modulation Optical Channels
}

\author{
Anas Chaaban, Senior Member, IEEE, Zouheir Rezki, Senior Member, IEEE, and Mohamed-Slim Alouini, Fellow, \\ IEEE
}

\begin{abstract}
The capacity of the intensity modulation direct detection multiple-input multiple-output channel is studied. Therein, the nonnegativity constraint of the transmit signal limits the applicability of classical schemes, including precoding. Thus, new ways are required for deriving capacity bounds for this channel. To this end, capacity lower bounds are developed in this paper by deriving the achievable rates of two precodingfree schemes: Channel inversion and QR decomposition. The achievable rate of a DC-offset SVD-based scheme is also derived as a benchmark. Then, capacity upper bounds are derived and compared against the lower bounds. As a result, the capacity at high signal-to-noise ratio (SNR) is characterized for the case where the number of transmit apertures is not larger than the number of receive apertures, and is shown to be achievable by the QR decomposition scheme. This is shown for a channel with average intensity or peak intensity constraints. Under both constraints, the high-SNR capacity is approximated within a small gap. Extensions to a channel with more transmit apertures than receive apertures are discussed, and capacity bounds for this case are derived.
\end{abstract}

\section{INTRODUCTION}

Incoherent transmission using intensity-modulation and direct-detection (IM-DD) is a simple technique where information is carried from a source to a destination by the signal intensity. Its simplicity makes it suitable for low cost/complexity optical-wireless communications (OWC). In this context, the receiver detects the modulated optical intensity using a photodiode.

The interest in IM-DD and its performance limits has increased significantly during this decade due to the revival of OWC [2] in general, and visible-light communication (VLC) [3] in particular. For surveys of recent advances in this field, the reader is referred to [2]-[7]. The focus of this paper is on multi-aperture IM-DD, where both the transmitter and the receiver potentially have multiple apertures. This forms an IMDD multiple input multiple output (MIMO) system, which has also been studied in the literature. For example, the benefits of MIMO for IM-DD OWC performance has been explored in [8]-[10] for on-off keying (OOK) and pulse-position modulation systems, respectively. The V-BLAST architecture [11]

A. Chaaban is with the School of Engineering, University of British Columbia, Kelowna, BC Canada V1Y 1V7. Email: anas.chaaban@ubc.ca.

Z. Rezki is with the Electrical and Computer Engineering Department, University of Idaho, Moscow, ID 83844 USA. Email: zrezki@uidaho.edu. The work of Z. Rezki is supported in part by the Qatar National Research Fund (a member of Qatar Foundation) under Grant NPRP 9-077-2-036. The statements made herein are solely the responsibility of the authors.

M.-S. Alouini is with the Division of Computer, Electrical, and Mathematical Sciences and Engineering (CEMSE) at King Abdullah University of Science and Technology (KAUST), Thuwal 23955-6900, Saudi Arabia. Email: slim.alouini@kaust.edu.sa.

Part of the results of this paper appeared in ICC 2017 [1]. was investigated in OOK-based IM-DD MIMO OWC systems in [12], [13]. An index modulation and OFDM based scheme was proposed for IM-DD MIMO in [14]. Constellation design for MIMO VLC has been studied in [15]. Multiuser IM-DD MIMO for OWC has been studied in [16], and transmission techniques and their comparison for indoor IM-DD MIMO OWC have been investigated in [17]-[19]. These works mostly focus on the bit error rate (BER) and outage performance.

In addition to studying a specific scheme and its performance, it is important to understand the fundamental performance limits of the system without restriction to a given scheme. Such limits measure the inherent fundamental capabilities of the channel. The focus of this paper is on one such fundamental quantity which is the channel capacity. This measures the maximum amount of information that can be sent reliably over the channel per unit time. The capacity of MIMO OWC modeled as a MIMO Poisson channel was studied in [20], [21]. Herein, we focus on the IM-DD channel with independent Gaussian noise as in [22]-[24]. This model is suitable for OWC with strong ambient light and/or thermal noise [25].

Thus, we study the capacity of the Gaussian IM-DD MIMO channel, which models e.g. VLC systems with multiple light fixtures and multiple detectors [18], or RGB (red/green/blue) color-shift keying systems [26]. Both average and peak intensity constraints are considered. The main difference with a radio-frequency (RF) MIMO channel is in the input constraints manifested in nonnegativity and average constraints in the IMDD case as opposed to power constraints in the RF case. To the best of our knowledge, the capacity of this channel has not been studied earlier in the literature. The first results thereon were given in [1] and in a parallel and independent work in [27]. Since characterizing capacity turns out to be a difficult task for IM-DD channels (even for single-input single-output (SISO) ones [22]-[24], [28]), this paper alternatively derives bounds on the channel capacity, which are tight in a practical regime of operation.

To derive capacity lower bounds, we study the achievable rates of transmission schemes which transform the MIMO channel into a set of parallel channels. Due to the IM-DD operation, the elegant singular-value decomposition (SVD) scheme used to transform RF MIMO channels to parallel channels can only be applied with some modifications. For example, a DC-offset can be used to conveniently produce a nonnegative signal [29]. This simultaneously imposes a constraint on the input alphabet [30], leading to a loss in the achievable rate. Fortunately, this loss is avoidable by using precoding-free schemes which rely on postcoding instead, such 
as channel inversion (CI) [17] or QR decomposition [31]. We derive the achievable rates of those schemes and their asymptotic performance at high SNR, which is the regime of operation of many OWC systems (cf. [32] e.g.).

To assess the performance of those schemes, we derive two capacity upper bounds. One is derived by representing the channel as a set of parallel SISO channels [33], [34], and the other by representing it as a set of parallel multiple-input single-output (MISO) channels (similar to [35, Sec. 4.1.1]). By comparing the asymptotic behavior of the first bound with that of the QR scheme under a total average intensity constraint, we characterize the high-SNR capacity of the channel under the condition $n_{\mathrm{t}} \leq n_{\mathrm{r}}$, where $n_{\mathrm{t}}$ and $n_{\mathrm{r}}$ are the numbers of transmit and receive apertures, respectively. Consequently, we show that a QR scheme, combined with an exponential input distribution and equal intensity allocation across the transmit apertures is high-SNR optimal. Furthermore, we derive the gap-to-optimality at high SNR of the CI and the DC-offset SVD schemes. The second upper bound complements the first one as it is tighter at lower SNR.

We also extend these results to the channel under peak intensity constraints, and we characterize the high-SNR capacity within a small gap under both average and peak constraints. Moreover, for the channel with $n_{\mathrm{t}}>n_{\mathrm{r}}$, we derive capacity upper bounds using a parallel MISO approach and lower bounds using precoding, QR-decomposition, and intensity allocation. The bounds do not coincide at high SNR but rather exhibit a constant gap. This highlights an interesting problem for future research. The gap vanishes for the MISO channel with an average constraint only, thus reproducing a result from [27]. To put this work in context with [27], Table I summarizes their differences and similarities. In general, our approach provides more engineering insight due to the way the schemes are constructed. In particular, the QR scheme achieves higher rates at moderate SNR for the MIMO channel with $n_{\mathrm{t}} \leq n_{\mathrm{r}}$ than the scheme in [27], since the former allows explicit intensity allocation which improves its performance at moderate SNR, while the latter uses the entropy-power inequality which is suited for high-SNR.

The paper is organized as follows. The channel model is introduced in Sec. II. Then the results related to the channel with a total average intensity constraints are given in Sec. III and proved in Sec. IV and V. The results are extended to the case under both average and peak constraints, and to the case with $n_{\mathrm{t}}>n_{\mathrm{r}}$ in Sec. VI. The paper is concluded in Sec. VII.

Notations: Throughout the paper, we use lowercase normal and boldface letters to denote deterministic scalars $(x)$ and vectors $(\boldsymbol{x})$, uppercase normal and boldface letters to denote random scalars $(X)$ and vectors $(\boldsymbol{X})$, and blackboard uppercase letters and calligraphic letters to denote matrices $(\mathbb{X})$ and sets $(\mathcal{X})$, respectively. The component of $\mathbb{X}$ in the $i^{\text {th }}$ row and $j^{\text {th }}$ column is denoted $x_{i, j}$. We denote the set of real numbers by $\mathcal{R}$ and the set of real nonnegative numbers by $\mathcal{R}_{+}$. We write $\mathbf{0}_{n}$ and $\mathbb{I}_{n}$ to denote an $n$-dimensional zero vector and an $n \times n$ identity matrix, $\mathbb{X}^{\top}$ and $\mathbb{X}^{-1}$ to denote the transpose and matrix inverse, and $\|\boldsymbol{x}\|$ and $\|\boldsymbol{x}\|_{1}$ to denote the $\ell_{2}$ and $\ell_{1}$ norms of a vector $\boldsymbol{x}$, respectively. We use $o(g(x))$ to denote a function $h(x)$ satisfying $\lim _{x \rightarrow \infty} \frac{h(x)}{g(x)}=0$. We write
TABLE I: Comparison with [27].

\begin{tabular}{|c|c|c|c|c|}
\hline \multicolumn{3}{|c|}{ Case } & [27] & This paper \\
\hline \multirow{3}{*}{ 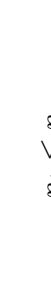 } & & $\begin{array}{l}\text { Upper } \\
\text { bound }\end{array}$ & $\begin{array}{c}\text { Duality } \\
\text { approach [36] }\end{array}$ & $\begin{array}{l}\text { Parallel SISO/MISO } \\
\text { approach }\end{array}$ \\
\hline & & $\begin{array}{l}\text { Lower } \\
\text { bound }\end{array}$ & $\begin{array}{l}\text { Entropy-power } \\
\text { inequality }\end{array}$ & $\begin{array}{l}\text { QR-decomposition \& } \\
\text { intensity allocation }\end{array}$ \\
\hline & & $\begin{array}{l}\text { Asymptotic } \\
\text { Capacity }\end{array}$ & $\begin{array}{l}\text { Peak, average, } \\
\text { \& combined } \\
\text { constraints }\end{array}$ & $\begin{array}{c}\text { Peak or average } \\
\text { constraints: Exact } \\
\text { Combined } \\
\text { constraints: Small gap }\end{array}$ \\
\hline \multirow{4}{*}{$\begin{array}{l}\hat{\varepsilon} \\
\hat{\Sigma}\end{array}$} & \multirow{3}{*}{$\begin{aligned} \stackrel{\wedge}{\wedge} \\
\xi\end{aligned}$} & $\begin{array}{l}\text { Upper } \\
\text { bound }\end{array}$ & - & $\begin{array}{l}\text { Parallel MISO } \\
\text { approach }\end{array}$ \\
\hline & & $\begin{array}{l}\text { Lower } \\
\text { bound }\end{array}$ & - & $\begin{array}{c}\text { Precoding, } \\
\text { QR-decomposition \& } \\
\text { intensity allocation }\end{array}$ \\
\hline & & $\begin{array}{l}\text { Asymptotic } \\
\text { Capacity }\end{array}$ & - & Constant gap \\
\hline & $\vec{n}$ & $\begin{array}{l}\text { Asymptotic } \\
\text { Capacity }\end{array}$ & $\begin{array}{c}\text { Peak, average, \& } \\
\text { combined constraints } \\
\text { (completed in [37]) }\end{array}$ & Average constraint \\
\hline
\end{tabular}

$\boldsymbol{X} \sim f(\boldsymbol{x})$ to indicate that $\boldsymbol{X}$ follows a distribution $f(\boldsymbol{x})$ and we write $\mathbf{N}\left(\mathbf{0}_{n}, \mathbb{Q}\right)$ to denote an $n$-dimensional Gaussian distribution with mean $\mathbf{0}_{n}$ and covariance matrix $\mathbb{Q}$. We denote the expectation, differential entropy, and mutual information by $\mathrm{E}[X], \mathrm{h}(X)$ and $\mathrm{I}(X ; Y)$, respectively.

\section{Channel Model}

Consider an OWC system comprising $n_{\mathrm{t}}$ transmit and $n_{\mathrm{r}}$ receive apertures, employing IM-DD. Denote the light intensity of the $i^{\text {th }}$ transmitter at time instant $k$ by $x_{i}(k) \in \mathcal{R}_{+}$. The received signal can be expressed in terms of $\boldsymbol{x}(k)=$ $\left[x_{1}(k), \cdots, x_{n_{\mathrm{t}}}(k)\right]^{\top}$ as (Fig. 1)

$$
\boldsymbol{Y}(k)=\mathbb{H} \boldsymbol{x}(k)+\boldsymbol{Z}(k), \quad k=1,2, \ldots
$$

where $\boldsymbol{Z}(k) \sim \mathrm{N}\left(\mathbf{0}_{n_{\mathrm{r}}}, \mathbb{I}_{n_{\mathrm{r}}}\right)$ is independent and identically distributed (i.i.d.) noise, ${ }^{1}$ and $\mathbb{H} \in \mathcal{R}_{+}^{n_{\mathrm{r}} \times n_{\mathrm{t}}}$ is the channel matrix with $h_{j, i}$ denoting the channel gain from transmitter $i$ to receiver $j$. This Gaussian noise model is common in the OWC context.

OWC channels tend to be static in indoor applications without mobility, or slowly varying with mobility or with scintillation and pointing error effects in outdoor applications [2], [38]. Due to this, one can assume that $\mathbb{H}$ remains fixed throughout a transmission duration. We further assume the availability of CSI at the receiver (CSIR) and the transmitter (CSIT), which can be obtained using channel estimation and feedback, respectively. The rank of $\mathbb{H}$ is assumed to be $\min \left\{n_{\mathrm{t}}, n_{\mathrm{r}}\right\}$. This holds with high probability, using proper spacing between transmit and receive apertures [18] or using angle/mirror diversity receivers [39] for instance.

The signal $x_{i}(k)$ is a realization of a random variable $X_{i}$ which satisfies

$$
X_{i} \geq 0, \text { and }\|\mathrm{E}[\boldsymbol{X}]\|_{1} \leq p_{\mathrm{o}},
$$

where $p_{\mathrm{o}}$ denotes the total optical power. We denote $\mathrm{E}\left[X_{i}\right]$ by $\xi_{i}$ and $\left[\xi_{1}, \ldots, \xi_{n_{\mathrm{t}}}\right]^{\top}$ by $\boldsymbol{\xi}$ throughout the paper. The second

\footnotetext{
${ }^{1}$ Correlated noise $\mathbf{N}\left(\mathbf{0}_{n_{\mathrm{r}}}, \mathbb{Q}\right)$ with invertible $\mathbb{Q}$ can be decorrelated at the receiver by multiplying the received signal by $\mathbb{Q}^{-1}$.
} 


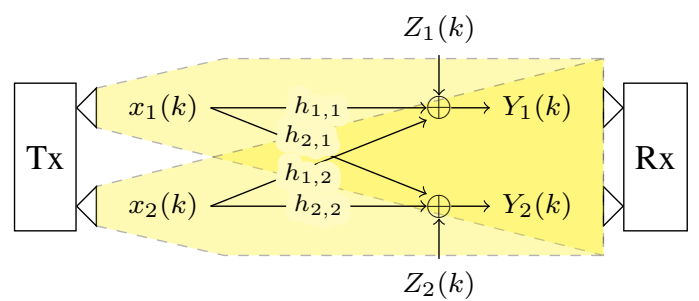

Fig. 1: A MIMO optical wireless communication system at time instant $k: x_{i}(k) \geq 0$ is the optical intensity, $h_{i, j} \geq 0$ is a channel gain, and $z_{i}(k)$ is Gaussian noise.

constraint is a total average intensity constraint, such as a lighting constraint in a VLC system. ${ }^{2}$ We call this channel an average-constrained IM-DD MIMO channel. The input may be subject to a peak constraint $X_{i} \leq x_{\max }$, which is considered in Sec. VI-A.

The capacity of this channel is defined in the standard Shannon sense [40], and is denoted by $c\left(\mathbb{H}, p_{\mathrm{o}}\right)$. This capacity is in particular given by $c\left(\mathbb{H}, p_{\mathrm{o}}\right)=\max _{f(\boldsymbol{x})} \mathrm{I}(\boldsymbol{X} ; \mathbb{H} \boldsymbol{X}+\boldsymbol{Z})$, where the maximization is with respect to probability laws of $\boldsymbol{X}$ that satisfy the constraints (2). This is indeed the largest transmission rate (in bits or nats per transmission) under which reliable communication is possible.

To study this capacity, we first describe the achievable rates of some practical transmission schemes. Then, we derive capacity upper bounds. Then, we assess the performance of the schemes by comparing their achievable rates against the upper bounds, which leads to the high-SNR capacity. Since the approach we present next is most suitable for the case $n_{\mathrm{t}} \leq n_{\mathrm{r}}$, we focus on this case henceforth. We shall comment on the case $n_{\mathrm{t}}>n_{\mathrm{r}}$ in Sec. VI.

\section{MAIN RESULTS FOR $n_{\mathrm{t}} \leq n_{\mathrm{r}}$}

\section{A. Achievable Rates}

Precoding and postcoding are common techniques in MIMO transmission. In RF MIMO channels, SVD provides optimal precoders and postcoders. Therein, the transmit signal is constructed as $\boldsymbol{x}(k)=\mathbb{V} \boldsymbol{s}(k)$, where $\boldsymbol{s}(k)$ is a codeword symbol from some alphabet $\mathcal{S} \subset \mathcal{R}^{n_{\mathrm{t}}}$, and $\mathbb{V} \in \mathcal{R}^{n_{\mathrm{t}} \times n_{\mathrm{t}}}$ is the right-singular matrix of $\mathbb{H}$. In the IM-DD case, the nonnegativity constraint on $\boldsymbol{x}(k)$ poses the following challenge: $\mathbb{V} \boldsymbol{s}(k) \in \mathcal{R}_{+}^{n_{\mathrm{t}}}$ must be satisfied for any $\boldsymbol{s}(k) \in \mathcal{S}$. Choosing nonnegative $s(k)$ (component-wise) does not suffice to make $\boldsymbol{x}(k)$ nonnegative. This forces an additional constraint on the alphabet $\mathcal{S}$, which now depends on $\mathbb{V}$ (cf. [30]).

This is not convenient in practice, where one is interested in designing a general codebook which is suitable for any $\mathbb{H}$. To alleviate this restriction, one can either refrain from precoding and rely on postcoding, or apply a DC-offset SVD scheme. Following this reasoning, next we present the achievable rates of two precoding-free schemes: Channel inversion (CI) and QR-decomposition (QR), followed by a precoding-based DCoffset SVD (DC-SVD) scheme.

\footnotetext{
${ }^{2}$ This is in contrast with the RF Gaussian MIMO channel where $X_{i}$ and $h_{j, i}$ are complex and $\sum_{i=1}^{n_{\mathrm{t}}} \mathrm{E}\left[\left|X_{i}\right|^{2}\right] \leq p$.
}

1) Precoding-free Schemes: To express the achievable rates of the CI and QR schemes, we need some prerequisites.

Definition 1: An average-constrained IM-DD SISO channel is one with input $x \in \mathcal{R}_{+}$satisfying $\mathrm{E}[X] \leq p_{\mathrm{o}}$, and output $Y=h x+Z$ where $h \in \mathcal{R}_{+}$and $Z \sim \mathrm{N}(0,1)$.

Lemma 1: In an average-constrained IM-DD SISO channel (Definition 1), the rates

$$
\begin{aligned}
& r_{\mathrm{e}}\left(h, p_{\mathrm{o}}\right)=\frac{1}{2} \log \left(1+\frac{e h^{2} p_{\mathrm{o}}^{2}}{2 \pi}\right), \text { and } \\
& r_{\mathrm{g}}\left(h, p_{\mathrm{o}}\right)=\max _{\ell>0} \mathrm{I}\left(X_{\mathrm{g}, p_{\mathrm{o}}}^{[\ell]} ; h X_{\mathrm{g}, p_{\mathrm{o}}}^{[\ell]}+Z\right),
\end{aligned}
$$

are achievable using $X \sim \frac{1}{p_{0}} e^{-\frac{x}{p_{0}}}$ (exponential distribution) [22], and $X=X_{\mathrm{g}, p_{\mathrm{o}}}^{[\ell]} \sim f_{\mathrm{g}, p_{\mathrm{o}}}^{[\ell]_{\mathrm{o}}}(x)=\sum_{k=0}^{\infty} \frac{\ell}{\ell+p_{\mathrm{o}}}\left(\frac{p_{\mathrm{o}}}{\ell+p_{\mathrm{o}}}\right)^{k} \delta(x-$ $k \ell$ ) (geometric distribution) [23], respectively, where $\delta(x)$ is the Dirac delta.

The CI and QR schemes transform the MIMO channel to a set of parallel channels of the form given in Definition 1 . In the CI scheme, this is done by multiplying $\boldsymbol{Y}(k)$ with the pseudo-inverse of $\mathbb{H}$ and then using a code for parallel SISO channels. This leads to the following statement.

Proposition 1: In an average-constrained IM-DD MIMO channel with $n_{\mathrm{t}} \leq n_{\mathrm{r}}$, CI achieves

$$
r_{\mathrm{m}}^{\mathrm{CI}}\left(\mathbb{H}, p_{\mathrm{o}}\right)=\max _{\boldsymbol{\xi} \in \mathcal{I}} \sum_{i=1}^{n_{\mathrm{t}}} r_{\mathrm{m}}\left(\left\|\boldsymbol{t}_{i}\right\|^{-1}, \xi_{i}\right), \quad \mathrm{m} \in\{\mathrm{e}, \mathrm{g}\},
$$

where $\boldsymbol{t}_{i}^{\top}$ is the $i^{\text {th }}$ row of $\mathbb{T}=\left[\mathbb{H}^{\top} \mathbb{H}\right]^{-1} \mathbb{H}^{\top} \in \mathcal{R}^{n_{\mathrm{t}} \times n_{\mathrm{r}}}$, and

$$
\mathcal{I}=\left\{\boldsymbol{\xi} \in \mathcal{R}_{+}^{n_{\mathrm{t}}} \mid\|\boldsymbol{\xi}\|_{1} \leq p_{\mathrm{o}}\right\} .
$$

Proof: The proof is given in Sec. IV-A1.

Note that $\mathbb{T}$ exists due to the assumption that $\mathbb{H}$ has rank $\min \left\{n_{\mathrm{t}}, n_{\mathrm{r}}\right\}=n_{\mathrm{t}}$ in this case.

Remark 1: This scheme does not require full CSIT. Feedback of $\left[\left\|t_{1}\right\|, \ldots,\left\|t_{M}\right\|\right]$ suffices.

Setting $\mathrm{m}=\mathrm{e}$ in (5), the optimal intensity allocation $\boldsymbol{\xi}$ satisfies

$$
\xi_{i} \in\left\{0, \frac{1}{2 \lambda} \pm \sqrt{\frac{1}{4 \lambda^{2}}-\frac{1}{c_{i}^{2}}}\right\}
$$

where $c_{i}=\left\|\boldsymbol{t}_{i}\right\|^{-1} \sqrt{\frac{e}{2 \pi}}$, and $\lambda>0$ is chosen so that $\|\boldsymbol{\xi}\|_{1}=$ $p_{\mathrm{o}}$. A reliable approximate solution can be obtained using the JA algorithm in [33]. This can also be used for $m=g$.

From Proposition 1, we can obtain the following corollary. Corollary 1: The achievable rate $r_{\mathrm{e}}^{\mathrm{CI}}\left(\mathbb{H}, p_{\mathrm{o}}\right)$ satisfies

$$
\lim _{p_{\mathrm{o}} \rightarrow \infty}\left(r_{\mathrm{e}}^{\mathrm{CI}}\left(\mathbb{H}, p_{\mathrm{o}}\right)-\sum_{i=1}^{n_{\mathrm{t}}} \frac{1}{2} \log \left(\frac{e p_{\mathrm{o}}^{2}}{2 \pi\left\|\boldsymbol{t}_{i}\right\|^{2} n_{\mathrm{t}}^{2}}\right)\right)=0 .
$$

Proof: The proof is given in Sec. IV-A2.

In the QR scheme, $\boldsymbol{Y}(k)$ is multiplied by an orthogonal matrix $\mathbb{Q}$ corresponding to the $\mathrm{QR}$ decomposition of the channel $\mathbb{H}=\mathbb{Q R}$ with $\mathbb{R}$ being an $n_{\mathrm{r}} \times n_{\mathrm{t}}$ upper triangular matrix. Decoding proceeds successively over the obtained triangular channel leading to the following.

Proposition 2: In an average-constrained IM-DD MIMO channel with $n_{\mathrm{t}} \leq n_{\mathrm{r}}$, a QR scheme achieves

$$
r_{\mathrm{m}}^{\mathrm{QR}}\left(\mathbb{H}, p_{\mathrm{o}}\right)=\max _{\mathbb{P} \in \mathcal{P}, \boldsymbol{\xi} \in \mathcal{I}} \sum_{i=1}^{n_{\mathrm{t}}} r_{\mathrm{m}}\left(\left|r_{i, i}^{[\mathbb{P}]}\right|, \xi_{\pi_{i}}\right), \quad \mathrm{m} \in\{\mathrm{e}, \mathrm{g}\},
$$


where $\mathcal{I}$ is defined in (6), $\mathcal{P}$ is the set of $n_{\mathrm{t}} \times n_{\mathrm{t}}$ permutation matrices, $\left[\pi_{1}, \ldots, \pi_{n_{\mathrm{t}}}\right]^{\top}=\mathbb{P}\left[1, \ldots, n_{\mathrm{t}}\right]^{\top}$, and $r_{i, i}^{[\mathbb{P}]}$ is a diagonal component of $\mathbb{R}^{[\mathbb{P}]}$ obtained from the QR decomposition of $\mathbb{H} \mathbb{P}$.

Proof: Details are given in Sec. IV-B1.

Here, $\mathbb{P}$ defines the decoding order of the $\mathrm{QR}$ scheme.

Remark 2: The QR scheme requires the feedback of $\left[r_{1,1}^{[\mathbb{P}]}, \ldots, r_{n_{\mathrm{t}}, n_{\mathrm{t}}}^{[\mathbb{P}]}\right]$ only.

For $\mathrm{m}=\mathrm{e}$ and a given $\mathbb{P}$, the optimal $\boldsymbol{\xi}$ is obtained by replacing $c_{i}$ in (7) with $\left|r_{i, i}^{[\mathbb{P}]}\right| \sqrt{\frac{e}{2 \pi}}$. This can also be used for $\mathrm{m}=\mathrm{g}$. The optimization with respect to the decoding order is more involved due to its discrete nature and coupling with $\boldsymbol{\xi}$. However, this optimization is not necessary at high SNR for $\mathrm{m}=\mathrm{e}$, where any $\mathbb{P} \in \mathcal{P}$ achieves the rate given next.

Corollary 2: The achievable rate $r_{\mathrm{e}}^{\mathrm{QR}}\left(\mathbb{H}, p_{\mathrm{o}}\right)$ satisfies

$$
\lim _{p_{\mathrm{o}} \rightarrow \infty}\left(r_{\mathrm{e}}^{\mathrm{QR}}\left(\mathbb{H}, p_{\mathrm{o}}\right)-\frac{1}{2} \log \left|\frac{e p_{\mathrm{o}}^{2}}{2 \pi n_{\mathrm{t}}^{2}} \mathbb{H}^{\top} \mathbb{H}\right|\right)=0 .
$$

Proof: See Sec. IV-B2.

Next, we present the achievable rate of the precoding-based DC-SVD scheme.

2) A Precoding-based Scheme: The advantage of DC-offset schemes is that they allow using practical alphabets for the codeword symbols such as binary phase-shift keying (BPSK), pulse-amplitude modulation (PAM), or more generally, any $s_{i}(k)$ chosen as a random variable $S_{i} \in\left[-a_{i}, a_{i}\right]$ for some $a_{i} \in \mathcal{R}_{+}$with $\mathrm{E}\left[S_{i}\right]=0$. A DC-offset takes care of the nonnegativity of the transmit signal in this case. Over a SISO channel, such a scheme achieves the following rates.

Lemma 2: In an average-constrained IM-DD SISO channel (Definition 1), the rates

$$
\begin{aligned}
& r_{\mathrm{u}}\left(h, p_{\mathrm{o}}\right)=\frac{1}{2} \log \left(1+\frac{2 h^{2} p_{\mathrm{o}}^{2}}{\pi e}\right), \text { and } \\
& r_{\mathrm{p}}\left(h, p_{\mathrm{o}}\right)=\max _{k>1} \mathrm{I}\left(X_{\mathrm{p}, p_{\mathrm{o}}}^{[k]} ; h X_{\mathrm{p}, p_{\mathrm{o}}}^{[k]}+Z\right),
\end{aligned}
$$

are achievable using $X=p_{\mathrm{o}}+S$ with $S$ uniform on $\left[-p_{\mathrm{o}}, p_{\mathrm{o}}\right]$ [22], and $X=X_{\mathrm{p}, p_{\mathrm{o}}}^{[k]}=p_{\mathrm{o}}+S_{\mathrm{p}, p_{\mathrm{o}}}^{[k]}$ where $S_{\mathrm{p}, p_{\mathrm{o}}}^{[k]} \sim f_{\mathrm{p}, p_{\mathrm{o}}}^{[k]}(s)=$ $\sum_{i=0}^{k-1} \frac{1}{k} \delta\left(s+p_{\mathrm{o}}-i \frac{2 p_{\mathrm{o}}}{k-1}\right)$ ( $k$-PAM) [41], respectively.

Note that the rate $r_{\mathrm{u}}\left(h, p_{\mathrm{o}}\right)$ is smaller than $r_{\mathrm{e}}\left(h, p_{\mathrm{o}}\right)$ given in Lemma 1. Nevertheless, this rate is useful for studying DCoffset schemes with a bounded input $X$ which is practical.

Lemma 2 is suitable for an SVD precoding scheme. Let the SVD of $\mathbb{H}$ be

$$
\mathbb{H}=\mathbb{U} \mathbb{B V} \mathbb{V}^{\top},
$$

where $\mathbb{U} \in \mathcal{R}^{n_{\mathrm{r}} \times n_{\mathrm{r}}}$ and $\mathbb{V} \in \mathcal{R}^{n_{\mathrm{t}} \times n_{\mathrm{t}}}$ are orthogonal matrices and $\mathbb{B} \in \mathcal{R}^{n_{\mathrm{r}} \times n_{\mathrm{t}}}$ is a diagonal matrix, i.e., $b_{i, j}=0 \forall i \neq j$. An achievable rate using the DC-SVD scheme is given next.

Proposition 3: In an average-constrained IM-DD MIMO channel with $n_{\mathrm{t}} \leq n_{\mathrm{r}}$, DC-SVD achieves

$$
r_{\mathrm{m}}^{\mathrm{SVD}}\left(\mathbb{H}, p_{\mathrm{o}}\right)=\max _{\boldsymbol{a} \in \mathcal{T}} \sum_{i=1}^{n_{\mathrm{t}}} r_{\mathrm{m}}\left(b_{i, i}, a_{i}\right), \quad \mathrm{m} \in\{\mathrm{u}, \mathrm{p}\}
$$

where $\mathcal{T}=\left\{\boldsymbol{a} \in \mathcal{R}_{+}^{n_{\mathrm{t}}}\left|\sum_{i=1}^{n_{\mathrm{t}}} \sum_{j=1}^{n_{\mathrm{t}}}\right| v_{i, j} \mid a_{j} \leq p_{\mathrm{o}}\right\}, \mathbb{B}$ is defined in (12), and $\mathbb{V}$ defined in (12).
Proof: The proof is given in Sec. IV-C1.

Remark 3: Contrary to the CI and QR schemes, the DCSVD scheme needs full CSIT knowledge (knowledge of $\mathbb{H}$ ) since the transmitter needs to know $\mathbb{V}$ and the optimal $\boldsymbol{a}$.

It remains to find the optimal $\boldsymbol{a}$ in Proposition 3. This optimization is different from the optimization with respect to $\xi$ in Propositions 1 and 2 due to the different feasible sets $\mathcal{I}$ and $\mathcal{T}$. The optimal $\boldsymbol{a}$ for $\mathrm{m}=\mathrm{u}$ can be derived similar to [33], and it satisfies

$$
a_{i} \nu_{i}=\in\left\{0, \frac{1}{2 \lambda} \pm \sqrt{\frac{1}{4 \lambda^{2}}-\frac{\nu_{i}^{2}}{c_{i}^{2}}}\right\}
$$

where $c_{i}=b_{i, i} \sqrt{\frac{2}{\pi e}}, \nu_{i}=\sum_{j=1}^{n_{\mathrm{t}}}\left|v_{j, i}\right|$, and $\lambda>0$ is chosen so that $\sum_{i=1}^{n_{\mathrm{t}}} a_{i} \nu_{i}=p_{\mathrm{o}}$. The algorithm in [33] can be used to obtain a reliable solution for $a_{i} \nu_{i}$ from which $a_{i}$ can be obtained. This allocation can be also used for $\mathrm{m}=\mathrm{p}$.

The asymptotic behavior of $r_{\mathrm{u}}^{\mathrm{SVD}}\left(\mathbb{H}, p_{\mathrm{o}}\right)$ is given next.

Corollary 3: The achievable rate $r_{\mathrm{u}}^{\mathrm{SVD}}\left(\mathbb{H}, p_{\mathrm{o}}\right)$ satisfies

$$
\lim _{p_{\mathrm{o}} \rightarrow \infty}\left(r_{\mathrm{u}}^{\mathrm{SVD}}\left(\mathbb{H}, p_{\mathrm{o}}\right)-\sum_{i=1}^{n_{\mathrm{t}}} \frac{1}{2} \log \left(\frac{2 b_{i, i}^{2} p_{\mathrm{o}}^{2}}{\pi e \nu_{i}^{2} n_{\mathrm{t}}^{2}}\right)\right)=0 .
$$

Proof: See Sec. IV-C2.

Next, we present capacity upper bounds useful for assessing the performance of these schemes.

\section{B. Capacity Upper Bounds}

We derive a capacity upper bound using a similar methodology as above. That is, we represent the capacity of the averageconstrained IM-DD MIMO channel in terms of the capacity of a system of parallel SISO or MISO channels. Then, we derive a capacity upper bound for the MIMO channel in terms of the resulting parallel channels. This leads to the following statement.

Theorem 1: The capacity of an average-constrained IM-DD MIMO channel with a channel matrix $\mathbb{H} \in \mathcal{R}_{+}^{n_{\mathrm{r}} \times n_{\mathrm{t}}}$ with rank $n_{\mathrm{t}} \leq n_{\mathrm{r}}$ is upper bounded by

$$
\begin{aligned}
& \bar{c}_{1}\left(\mathbb{H}, p_{\mathrm{o}}\right)=\max _{\boldsymbol{\xi} \in \mathcal{I}} \sum_{i=1}^{n_{\mathrm{t}}} \bar{r}\left(k_{i, i}^{-\frac{1}{2}}, \xi_{i}\right)+\zeta, \text { and } \\
& \bar{c}_{2}\left(\mathbb{H}, p_{\mathrm{o}}\right)=\max _{\boldsymbol{\xi} \in \mathcal{I}} \sum_{i=1}^{n_{\mathrm{r}}} \bar{r}\left(1, \hat{\xi}_{i}\right),
\end{aligned}
$$

where $\zeta=\frac{1}{2} \log \left(\frac{\prod_{i=1}^{n_{\mathrm{t}}} k_{i, i}}{|\mathbb{K}|}\right), \mathbb{K}=\left[\mathbb{H}^{\top} \mathbb{H}\right]^{-1}$ and $k_{i, i}$ its diagonal component, $\mathcal{I}$ is defined in (6), $\left[\hat{\xi}_{1}, \ldots, \hat{\xi}_{n_{\mathrm{r}}}\right]^{\top}=\mathbb{H} \boldsymbol{\xi}$, and $\bar{r}(h, \xi)=\inf _{\beta, \delta>0} b(h \xi, \beta, \delta)$ where $b(h \xi, \beta, \delta)$ is given in (18) at the bottom of next page. ${ }^{3}$

Proof: Details are given in Sec. V-A.

Note that $\bar{r}\left(h, p_{\mathrm{o}}\right)$ is an upper bound on the capacity of an average-constrained IM-DD SISO channel (Definition 1) [22]. Theorem 1 continues to holds if we replace $\bar{r}(\cdot, \cdot)$ with other upper bounds from [22]-[24]. The bound $\bar{c}_{1}\left(\mathbb{H}, p_{\mathrm{o}}\right)$ coincides with the high-SNR capacity of the MIMO channel as given next.

$$
{ }^{3} Q(x) \text { is the Q-function, i.e., } Q(x)=\frac{1}{\sqrt{2 \pi}} \int_{x}^{\infty} e^{-\frac{u^{2}}{2}} d u \text {. }
$$




\section{High-SNR Capacity}

The high-SNR capacity is characterized next.

Theorem 2: For an average-constrained IM-DD MIMO channel with a full column rank $\mathbb{H} \in \mathcal{R}_{+}^{n_{\mathrm{r}} \times n_{\mathrm{t}}}$, the capacity satisfies

$$
\lim _{p_{\mathrm{o}} \rightarrow \infty}\left(c\left(\mathbb{H}, p_{\mathrm{o}}\right)-\frac{1}{2} \log \left|\frac{e p_{\mathrm{o}}^{2}}{2 \pi n_{\mathrm{t}}^{2}} \mathbb{H}^{\mathrm{T}} \mathbb{H}\right|\right)=0,
$$

and is asymptotically achievable using the QR scheme.

Proof: This is obtained by comparing the high-SNR asymptote of the upper bound in Theorem 1 with Corollary 2 . Details are given in Sec. V-B.

This theorem implies that the transmitter has low complexity at high SNR, where no precoding is needed and equal intensity allocation is optimal. The same does not hold true for the DCSVD scheme, which in spite of intensity allocation in addition to precoding, is not optimal at high SNR as we shall see next.

\section{Comparison}

For comparison, we use the VLC scenario given in [18], in which a transmitter with 4 light fixtures communicates with a receiver with 4 detectors $\left(n_{\mathrm{t}}=n_{\mathrm{r}}=4\right)$. The details of the system are given in Table II for two receiver positions. The channel coefficients follow a Lambertian propagation pattern, leading to the matrices given in Table III (cf. [18, Eq. (14) and (18)]).

In Fig. 2, we plot the capacity upper bounds in Theorem 1 , in addition to the achievable rates $r_{\mathrm{m}}^{\mathrm{CI}}\left(\mathbb{H}, p_{\mathrm{o}}\right), r_{\mathrm{m}}^{\mathrm{QR}}\left(\mathbb{H}, p_{\mathrm{o}}\right)$, and $r_{\mathrm{m}}^{\mathrm{SVD}}\left(\mathbb{H}, p_{\mathrm{o}}\right)$ (Propositions $1-3$, respectively). These are plotted versus SNR, defined as the ratio $\frac{p_{\mathrm{o}}}{\sigma}$ where $\sigma^{2}=1$ is the noise variance at each aperture. The intensity allocation for the exponential (exp.) and the uniform (unif.) distributed inputs are obtained using the algorithm in [33], whereas the intensity allocation for the geometric (geom.) and PAM distributed inputs are obtained numerically using grid-search. In the same figure, $\bar{c}_{1}\left(\mathbb{H}, p_{\mathrm{o}}\right)$ and $\bar{c}_{2}\left(\mathbb{H}, p_{\mathrm{o}}\right)$ from Theorem 1 are plotted as upper bounds 1 and 2, respectively.

Fig. 2 shows that the QR scheme outperforms the DC-SVD scheme. The performance gap between these schemes is as large as $\approx 3.6 \mathrm{~dB}$ at high SNR in Fig. $2 \mathrm{a}$ and $\approx 4.2 \mathrm{~dB}$ in Fig. 2b. Note also that the QR scheme is better than the CI scheme, which agrees with intuition since CI amplifies noise contrary to the $\mathrm{QR}$ scheme. The gap between the two is $\approx$ $0.9 \mathrm{~dB}$ in Fig. $2 \mathrm{a}$ and $\approx 13.1 \mathrm{~dB}$ in Fig. $2 \mathrm{~b}$. The gap to highSNR capacity of CI and DC-SVD is calculated explicitly in Appendix $\mathrm{B}$, where it is shown that $\mathrm{CI}$ is only optimal if $\mathbb{H}$ has a parallel channel structure, in which case the gap of DCSVD is minimal. Finally, note that the QR/exp lower bound and the upper bound $\bar{c}_{1}\left(\mathbb{H}, p_{\mathrm{o}}\right)$ converge as SNR increases, demonstrating Theorem 2.

Fig. 2 also shows that the upper bound $\bar{c}_{1}\left(\mathbb{H}, p_{\mathrm{o}}\right)$ is loose at lower SNR. This upper bound is always larger than $\frac{1}{2} \log \left(\frac{\prod_{i=1}^{n_{t}} k_{i, i}}{|\mathbb{K}|}\right)$, which makes it not suitable for lower SNR. However, it is tight at high SNR, which is common in the OWC context ${ }^{4}$. The bound $\bar{c}_{2}\left(\mathbb{H}, p_{\mathrm{o}}\right)$ complements this bound as it is tighter at lower SNR. Table IV compares the schemes in terms of their CSIT requirements. The QR and CI schemes are superior in this aspect in comparison with the DC-SVD scheme (Remarks 1-3).

\section{TRANSMisSion SCHEMES FOR $n_{\mathrm{t}} \leq n_{\mathrm{r}}$}

We start by describing precoding-free schemes, whose advantage is that they allow using any nonnegative input without any restriction due to $\mathbb{H}$. They also have less CSIT requirements. A precoding-free scheme is based on sending $\boldsymbol{x}(k) \in$ $\mathcal{R}_{+}^{n_{\mathrm{t}}}$ at time $k$, where $x_{i}(k)$ is a symbol of a length- $l$ codeword $\left[x_{i}(1), \ldots, x_{i}(l)\right]$ encoded using an average-constrained IMDD SISO encoder. To decode all transmitted streams, i.e., $\left[x_{i}(1), \ldots, x_{i}(l)\right], i \in\left\{1, \ldots, n_{\mathrm{t}}\right\}$, the receiver uses postcoding by either channel inversion or QR decomposition to transform the channel into a set of parallel SISO channels. The details of these schemes are discussed next.

\section{A. Channel-Inversion Scheme}

1) Achievable Rate: In a channel-inversion (CI) scheme, the received signal is multiplied by the pseudo-inverse of $\mathbb{H}$ given by $\mathbb{T}=\left[\mathbb{H}^{\top} \mathbb{H}\right]^{-1} \mathbb{H}^{\top} \in \mathcal{R}^{n_{\mathrm{t}} \times n_{\mathrm{r}}}$, i.e., $\mathbb{T} \mathbb{H}=\mathbb{I}_{n_{\mathrm{t}}}$. This exists since $\operatorname{rank}(\mathbb{H})=\min \left\{n_{\mathrm{t}}, n_{\mathrm{r}}\right\}=n_{\mathrm{t}}$. After this multiplication, the receiver has

$$
\overline{\boldsymbol{Y}}(k)=\boldsymbol{x}(k)+\mathbb{T} \boldsymbol{Z}(k)=\boldsymbol{x}(k)+\overline{\boldsymbol{Z}}(k),
$$

for $k=1, \ldots, l$. This is a system of parallel channels with i.i.d. $N\left(\mathbb{I}_{n_{\mathrm{t}}}, \mathbb{T}^{\mathrm{T}}\right)$ spatially correlated noise. The receiver ignores this correlation and decodes $\left[x_{i}(1), \ldots, x_{i}(l)\right]$ from $\left[\bar{Y}_{i}(1), \ldots, \bar{Y}_{i}(l)\right]$, for $i \in\left\{1, \ldots, n_{\mathrm{t}}\right\}$. Since $\bar{Z}_{i}(k) \sim$ $\mathrm{N}\left(0,\left\|\boldsymbol{t}_{i}\right\|^{2}\right)$ (recall that $\boldsymbol{t}_{i}$ is the $i^{\text {th }}$ row of $\left.\mathbb{T}\right)$, the channel $\bar{Y}_{i}(k)=x_{i}(k)+\bar{Z}_{i}(k)$ is equivalent to an average-constrained IM-DD SISO channel (Definition 1), with $h=\left\|\boldsymbol{t}_{i}\right\|^{-1}$. According to Lemma 1 , the rate $r_{\mathrm{m}}\left(\left\|\boldsymbol{t}_{i}\right\|^{-1}, \xi_{i}\right), \mathrm{m} \in\{\mathrm{e}, \mathrm{g}\}$, is achievable over this channel, where $\xi_{i}$ is the average intensity allocated to the $i^{\text {th }}$ stream. Maximizing with respect to the intensity allocation $\boldsymbol{\xi}$ leads to the achievable rate

$$
r_{\mathrm{m}}^{\mathrm{CI}}\left(\mathbb{H}, p_{\mathrm{o}}\right)=\max _{\boldsymbol{\xi} \in \mathcal{I}} \sum_{i=1}^{n_{\mathrm{t}}} r_{\mathrm{m}}\left(\left\|\boldsymbol{t}_{i}\right\|^{-1}, \xi_{i}\right),
$$

with $\mathcal{I}$ as defined in Proposition 1. This proves this proposition.

${ }^{4}$ For instance, an optical intensity of $10 \mathrm{~W}$, photodetector responsivity of $0.6 \mathrm{~A} / \mathrm{W}$, and noise variance of $2 \times 10^{-14} \mathrm{~A}^{2}$ as in [32] leads to an SNR (excluding channel gain) in the order of $10^{7}$, i.e., $70 \mathrm{~dB}$.

$$
b(\xi, \beta, \delta)=\log \left(\frac{\beta e^{-\frac{\delta^{2}}{2}}}{\sqrt{2 \pi e}}+\frac{Q(\delta)}{\sqrt{e}}\right)+\frac{Q(\delta)+\delta^{2}(1-Q(\delta+\xi))}{2}+\frac{\delta+\xi}{\beta}+\frac{e^{-\frac{\delta^{2}}{2}}}{\sqrt{2 \pi}}\left(\frac{1}{\beta}+\frac{\delta}{2}\right)
$$


TABLE II: VLC system parameters of Fig. 2.

\begin{tabular}{l|c} 
Room dimensions & $4 \mathrm{~m} \times 4 \mathrm{~m}$ \\
\hline Tx positions & $(2 \pm 0.3,2 \pm 0.3,2.5) \mathrm{m}$ \\
\hline Rx positions: Case $\mathrm{a}$ & $(2 \pm 0.05,2 \pm 0.05,0.75) \mathrm{m}$ \\
\hline Rx positions: Case $\mathrm{b}$ & $(2.5 \pm 0.05,3 \pm 0.05,0.75) \mathrm{m}$ \\
\hline Tx semiangle & $15^{\circ}$ \\
\hline Rx semiangle & $15^{\circ}$ \\
\hline Detector area & $1 \mathrm{~cm}^{2}$
\end{tabular}

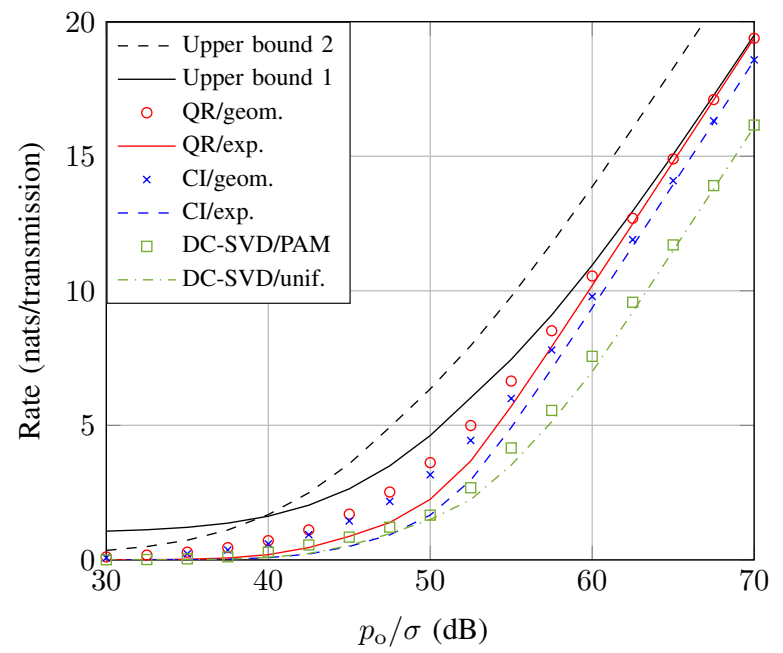

(a) Case a: Receiver centered at $(2,2,0.75) \mathrm{m}$.
TABLE III: Channel matrices of Fig. 2.

\begin{tabular}{c|ccccc} 
& & & \\
Case a & $\mathbb{H}_{\mathrm{a}}=10^{-4}\left[\begin{array}{cccc}.6888 & .5559 & .5559 & 0 \\
.5559 & .6888 & 0 & .5559 \\
.5559 & 0 & .6888 & .5559 \\
0 & .5559 & .5559 & .6888\end{array}\right]$ \\
\hline \multirow{3}{*}{ Case b } & $\mathbb{H}_{\mathrm{b}}=10^{-4}\left[\begin{array}{cccc}.0461 & .0272 & .0358 & .0213 \\
.2573 & .1798 & .1917 & .1352 \\
.0735 & .0424 & .0713 & .0412 \\
.4426 & .3040 & .4275 & .2940\end{array}\right]$
\end{tabular}

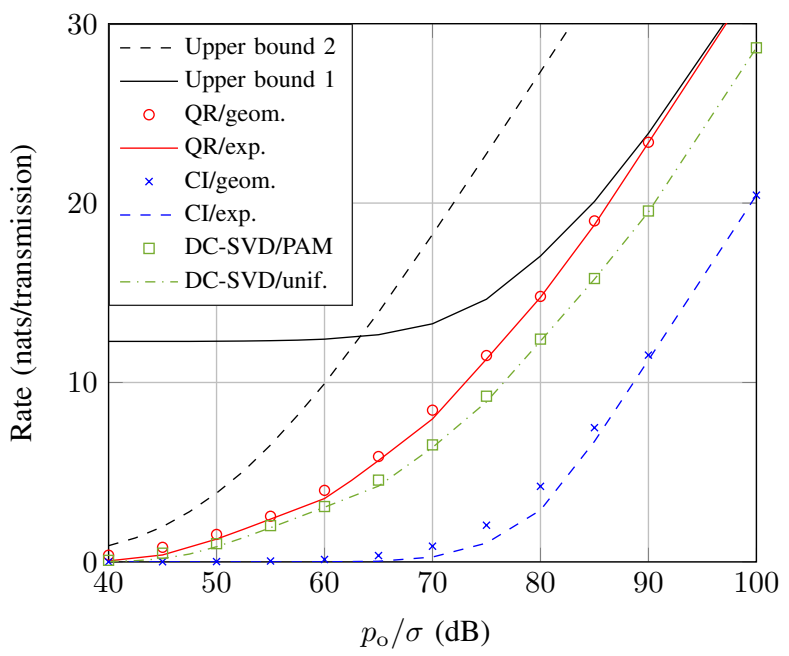

(b) Case b: Receiver centered at $(2.5,3,0.75) \mathrm{m}$.

Fig. 2: Achievable rates and upper bounds for the MIMO channel described in Table II.

TABLE IV: Required amount of feedback for each scheme.

\begin{tabular}{c||c|c|c} 
Scheme & $\mathrm{CI}$ & $\mathrm{QR}$ & DC-SVD \\
\hline Feedback (real scalars) & $n_{\mathrm{t}}$ & $n_{\mathrm{t}}$ & $n_{\mathrm{t}} n_{\mathrm{r}}$
\end{tabular}

2) High-SNR Asymptotic Rate: Letting $\mathrm{m}=\mathrm{e}$ leads to

$$
r_{\mathrm{e}}^{\mathrm{CI}}\left(\mathbb{H}, p_{\mathrm{o}}\right)=\max _{\boldsymbol{\xi} \in \mathcal{I}} \sum_{i=1}^{n_{\mathrm{t}}} \frac{1}{2} \log \left(1+\frac{e \xi_{i}^{2}}{2 \pi\left\|\boldsymbol{t}_{i}\right\|^{2}}\right) .
$$

This maximization is not a convex optimization problem, and its solution is not standard water-filling. This parallel channel intensity allocation problem was studied in [33]. Therein, it is shown that as $p_{\mathrm{o}}$ increases, the optimal intensity allocation approaches $\xi_{i}=\frac{p_{\mathrm{o}}}{n_{\mathrm{t}}} \forall i$. Thus, $r_{\mathrm{e}}^{\mathrm{CI}}\left(\mathbb{H}, p_{\mathrm{o}}\right) \rightarrow$ $\sum_{i=1}^{n_{\mathrm{t}}} \frac{1}{2} \log \left(\frac{e p_{\mathrm{o}}^{2}}{2 \pi\left\|\boldsymbol{t}_{i}\right\|^{2} n_{\mathrm{t}}^{2}}\right)$ as $p_{\mathrm{o}} \rightarrow \infty$, which proves Corollary 1 .

This achievable rate can be improved using a QRdecomposition receiver and successive decoding and cancellation as described next.

\section{B. QR-Decomposition Scheme}

1) Achievable Rate: In this case, the receiver employs $Q R$ decomposition to reduce the channel into a more desirable structure, where successive decoding of the $n_{\mathrm{t}}$ streams can be easily applied. Let the $\mathrm{QR}$ decomposition of $\mathbb{H}$ be $\mathbb{H}=\mathbb{Q R}$, where $\mathbb{Q} \in \mathcal{R}^{n_{\mathrm{r}} \times n_{\mathrm{r}}}$ is orthogonal and $\mathbb{R} \in \mathcal{R}^{n_{\mathrm{r}} \times n_{\mathrm{t}}}$ is upper triangular $\left(r_{i, j}=0\right.$ if $\left.i>j\right)$. Then, if the receiver multiplies $\boldsymbol{Y}(k)$ by $\mathbb{Q}^{\top}$, it obtains $\tilde{\boldsymbol{Y}}(k)=\mathbb{R} \boldsymbol{x}(k)+\tilde{\boldsymbol{Z}}(k)$. From this signal, it can start by decoding $\left[x_{n_{\mathrm{t}}}(1), \ldots, x_{n_{\mathrm{t}}}(l)\right]$ from $\left[\tilde{Y}_{n_{\mathrm{t}}}(1), \ldots, \tilde{Y}_{n_{\mathrm{t}}}(l)\right]$. This is a SISO channel since $\tilde{Y}_{n_{\mathrm{t}}}(k)=$ $r_{n_{\mathrm{t}}, n_{\mathrm{t}}} x_{n_{\mathrm{t}}}(k)+\tilde{Z}_{n_{\mathrm{t}}}(k)$. Then it subtracts $x_{n_{\mathrm{t}}}(k)$ from $\tilde{Y}_{n_{\mathrm{t}}-1}(k)$ to obtain $R_{n_{\mathrm{t}}-1, n_{\mathrm{t}}-1} x_{n_{\mathrm{t}}-1}(k)+\tilde{Z}_{n_{\mathrm{t}}-1}(k), k=1, \ldots, l$, and decodes $\left[x_{n_{\mathrm{t}}-1}(1), \ldots, x_{n_{\mathrm{t}}-1}(l)\right]$ and so on.

This way, the decoding order is $n_{\mathrm{t}}, n_{\mathrm{t}}-1, \ldots, 1$. Other decoding orders can be achieved using a permutation matrix. Fix $\mathbb{P}$ to be an $n_{\mathrm{t}} \times n_{\mathrm{t}}$ permutation matrix, and note that $\mathbb{P P}^{\top}=\mathbb{I}_{n_{\mathrm{t}}}$. Then,

$$
\begin{aligned}
\boldsymbol{Y}(k) & =\mathbb{H} \mathbb{P P}^{\top} \boldsymbol{x}(k)+\boldsymbol{Z}(k) \\
& =\mathbb{Q}^{[\mathbb{P}]} \mathbb{R}^{[\mathbb{P}]} \mathbb{P}^{\top} \boldsymbol{x}(k)+\boldsymbol{Z}(k),
\end{aligned}
$$

where $\mathbb{Q}^{[\mathbb{P}]} \mathbb{R}^{[\mathbb{P}]}$ is the $\mathrm{QR}$ decomposition of $\mathbb{H} \mathbb{P}$. Note that $\mathbb{P}^{\top} \boldsymbol{x}(k)$ is a permutation of the components of $\boldsymbol{x}(k)$ according to $\boldsymbol{\pi}=\mathbb{P}\left[1, \ldots, n_{\mathrm{t}}\right]^{\top}$, i.e., its first component is $x_{\pi_{1}}(k)$, its second is $x_{\pi_{2}}(k)$, and so on. The receiver multiplies $\boldsymbol{Y}(k)$ by $\mathbb{Q}^{[\mathbb{P}] \mathrm{T}}$ to obtain

$$
\tilde{\boldsymbol{Y}}(k)=\mathbb{R}^{[\mathbb{P}]} \mathbb{P}^{\top} \boldsymbol{x}(k)+\tilde{\boldsymbol{Z}}(k),
$$

where $\tilde{\boldsymbol{Z}}(k)=\mathbb{Q}^{[\mathbb{P}] T} \boldsymbol{Z}(k)$ is i.i.d. $\mathbf{N}\left(\mathbf{0}_{n_{\mathrm{r}}}, \mathbb{I}_{n_{\mathrm{r}}}\right)$. It starts by considering $\tilde{Y}_{n_{\mathrm{t}}}(k)=r_{n_{\mathrm{t}}, n_{\mathrm{t}}}^{[\mathbb{P}} x_{\pi_{n_{\mathrm{t}}}}(k)+\tilde{Z}_{n_{\mathrm{t}}}(k), k=$ $1, \ldots, l$. If $r_{n_{\mathrm{t}}, n_{\mathrm{t}}}^{[\mathbb{P}]}<0$, it multiplies $\tilde{Y}_{n_{\mathrm{t}}}(k)$ by -1 to obtain $\left|r_{n_{\mathrm{t}}, n_{\mathrm{t}}}^{[\mathbb{P}]}\right| x_{\pi_{n_{\mathrm{t}}}}(k)+\tilde{Z}_{n_{\mathrm{t}}}^{\prime}(k), k=1, \ldots, l$, where $\tilde{Z}_{n_{\mathrm{t}}}^{\prime}(k)$ is i.i.d. $\mathrm{N}(0,1)$. Then, it decodes $\left[x_{\pi_{n_{\mathrm{t}}}}(1), \ldots, x_{\pi_{n_{\mathrm{t}}}}(l)\right]$ achieving $r_{\mathrm{m}}\left(\left|r_{n_{\mathrm{t}}, n_{\mathrm{t}}}^{[\mathbb{P}]}\right|, \xi_{\pi_{n_{\mathrm{t}}}}\right), \mathrm{m} \in\{\mathrm{e}, \mathrm{g}\}$ (Lemma 1) where $\xi_{\pi_{n_{\mathrm{t}}}}$ is the average intensity allocated to $x_{\pi_{n_{t}}}(k)$. The receiver then subtracts the contribution of $x_{\pi_{n_{\mathrm{t}}}}(k)$ from $\tilde{Y}_{n_{\mathrm{t}}-1}(k)$ 
to obtain $r_{n_{\mathrm{t}}-1, n_{\mathrm{t}}-1}^{[\mathbb{P}]} x_{\pi_{n_{\mathrm{t}}-1}}(k)+\tilde{Z}_{n_{\mathrm{t}}-1}(k), k=1, \ldots, l$, from which it decodes $\left[x_{\pi_{n_{\mathrm{t}}-1}}(1), \ldots, x_{\pi_{n_{\mathrm{t}}-1}}(l)\right]$ achieving $r_{\mathrm{m}}\left(\left|r_{n_{\mathrm{t}}-1, n_{\mathrm{t}}-1}^{[\mathbb{P}]}\right|, \xi_{\pi_{n_{\mathrm{t}}-1}}\right)$. This proceeds until all $n_{\mathrm{t}}$ streams have been decoded following the order $\pi_{1}, \ldots, \pi_{n_{\mathrm{t}}}$. The total achievable rate is then optimized with respect to $\boldsymbol{\xi}$ and $\mathbb{P}$. This leads to the achievability of

$$
r_{\mathrm{m}}^{\mathrm{QR}}\left(\mathbb{H}, p_{\mathrm{o}}\right)=\max _{\mathbb{P} \in \mathcal{P}, \boldsymbol{\xi} \in \mathcal{I}} \sum_{i=1}^{n_{\mathrm{t}}} r_{\mathrm{m}}\left(\left|r_{i, i}^{[\mathbb{P}]}\right|, \xi_{\pi_{i}}\right),
$$

as given in Proposition 2, where $\mathcal{I}$ is defined in (6), $\mathcal{P}$ is the set of $n_{\mathrm{t}} \times n_{\mathrm{t}}$ permutation matrices.

2) High-SNR Asymptotic Rate: For $\mathrm{m}=\mathrm{e}$, the achievable rate above becomes

$$
r_{\mathrm{e}}^{\mathrm{QR}}\left(\mathbb{H}, p_{\mathrm{o}}\right)=\max _{\mathbb{P} \in \mathcal{P}} \max _{\xi \in \mathcal{I}} \sum_{i=1}^{n_{\mathrm{t}}} \frac{1}{2} \log \left(1+\frac{e\left(r_{i, i}^{[\mathbb{P}]} \xi_{\pi_{i}}\right)^{2}}{2 \pi}\right) .
$$

The inner maximization is an intensity allocation problem for a system of parallel channels, with channels $\left[\left|r_{1,1}^{[\mathbb{P}]}\right|, \ldots,\left|r_{n_{\mathrm{t}}, n_{\mathrm{t}}}^{[\mathbb{P}]}\right|\right]$, which has been studied in [33]. The optimal solution with respect to $\boldsymbol{\xi}$ approaches $\xi_{i}=\frac{p_{\mathrm{o}}}{n_{\mathrm{t}}}, \forall i$ as $p_{\mathrm{o}} \rightarrow \infty$ [33]. Thus, the achievable rate satisfies

$$
\begin{aligned}
& r_{\mathrm{e}}^{\mathrm{QR}}\left(\mathbb{H}, p_{\mathrm{o}}\right) \\
& =\max _{\mathbb{P} \in \mathcal{P}} \sum_{i=1}^{n_{\mathrm{t}}} \frac{1}{2} \log \left(\frac{e\left(r_{i, i}^{[\mathbb{P}]} p_{\mathrm{o}}\right)^{2}}{2 \pi n_{\mathrm{t}}^{2}}\right)+o\left(\log \left(p_{\mathrm{o}}\right)\right) \\
& =\max _{\mathbb{P} \in \mathcal{P}} \frac{1}{2} \log \left(\frac{\left(e p_{\mathrm{o}}^{2}\right)^{n_{\mathrm{t}}}}{\left(2 \pi n_{\mathrm{t}}^{2}\right)^{n_{\mathrm{t}}}} \prod_{i=1}^{n_{\mathrm{t}}}\left(r_{i, i}^{[\mathbb{P}]}\right)^{2}\right)+o\left(\log \left(p_{\mathrm{o}}\right)\right) .
\end{aligned}
$$

Since $\prod_{i=1}^{n_{\mathrm{t}}}\left(r_{i, i}^{[\mathbb{P}]}\right)^{2}=\left|\mathbb{H}^{\top} \mathbb{H}\right|$ for any $\mathbb{P}$, we conclude that $r_{\mathrm{e}}^{\mathrm{QR}}\left(\mathbb{H}, p_{\mathrm{o}}\right)=\frac{1}{2} \log \left|\frac{e p_{\mathrm{o}}^{2}}{2 \pi n_{\mathrm{t}}^{2}} \mathbb{H}^{\top} \mathbb{H}\right|+o\left(\log \left(p_{\mathrm{o}}\right)\right)$, which proves Corollary 2 . Note that the QR scheme significantly simplifies at high SNR, where its achievable rate is maximized by choosing $\mathbb{P}=\mathbb{I}_{n_{\mathrm{t}}}$ and $\xi_{i}=\frac{p_{\mathrm{o}}}{n_{\mathrm{t}}}, \forall i$.

\section{DC-offset SVD Scheme}

1) Achievable Rate: In a DC-SVD scheme, the right singular vectors of $\mathbb{H}$ are used for precoding at the transmitter. Let the SVD of $\mathbb{H}$ be $\mathbb{H}=\mathbb{U B V} \mathbb{V}^{\top}$. We construct the transmit signal as $\boldsymbol{x}(k)=\mathbb{V} \boldsymbol{s}(k)+\boldsymbol{t}$ where $s_{i}(k)$ is a realization of a random variable $S_{i} \in\left[-a_{i}, a_{i}\right]$ for some $a_{i} \in \mathcal{R}_{+}$ with $\mathrm{E}\left[S_{i}\right]=0$. Since the largest negative value of $x_{i}(k)$ is $-\sum_{j=1}^{n_{\mathrm{t}}}\left|v_{i, j}\right| a_{j}$, then, to guarantee nonnegativity, it is required that $t_{i}=\sum_{j=1}^{n_{\mathrm{t}}}\left|v_{i, j}\right| a_{j}$. Thus, $\mathrm{E}\left[X_{i}\right]=\sum_{j=1}^{n_{\mathrm{t}}}\left|v_{i, j}\right| a_{j}$.

Upon receiving $\boldsymbol{Y}(k)$, the receiver calculates $\mathbb{U}^{\top}(\boldsymbol{Y}(k)-$ $\mathbb{H} \boldsymbol{t})$ to obtain $\hat{\boldsymbol{Y}}(k)=\mathbb{B} \boldsymbol{s}(k)+\hat{\boldsymbol{Z}}(k), k=1, \ldots, l$, where $\hat{\boldsymbol{Z}}(k)$ is i.i.d. $\mathbf{N}\left(\mathbf{0}_{n_{\mathrm{r}}}, \mathbb{I}_{n_{\mathrm{r}}}\right)$. This is a parallel channel where $\hat{Y}_{i}(k)=b_{i, i} s_{i}(k)+\hat{Z}_{i}(k)$ for $i=1, \ldots, n_{\mathrm{t}}$ and $\hat{Y}_{i}(k)$ is noise otherwise $\left(\operatorname{rank}(\mathbb{H})=n_{\mathrm{t}} \leq n_{\mathrm{r}}\right)$. By Lemma 2, the achievable rate over channel $i$ is $r_{\mathrm{m}}\left(b_{i, i}, a_{i}\right), \mathrm{m} \in\{\mathrm{u}, \mathrm{p}\}$. The overall achievable rate is

$$
r_{\mathrm{m}}^{\mathrm{SVD}}\left(\mathbb{H}, p_{\mathrm{o}}\right)=\sum_{i=1}^{n_{\mathrm{t}}} r_{\mathrm{m}}\left(b_{i, i}, a_{i}\right),
$$

which is to be maximized with respect to $a_{i}$ subject to the total intensity constraint, i.e., $\sum_{i=1}^{n_{\mathrm{t}}} \sum_{j=1}^{n_{\mathrm{t}}}\left|v_{i, j}\right| a_{j} \leq p_{\mathrm{o}}$. This proves Proposition 3.

Note that due to precoding using $\mathbb{V}$, applying a DCoffset is necessary in this scheme. Therefore, the unbounded exponential and geometrically distributed inputs in Lemma 1 are not suitable for this scheme.

2) High-SNR Asymptotic Rate: The problem of allocating $a_{i}$ for $\mathrm{m}=\mathrm{u}$ can be written as:

$$
r_{\mathrm{u}}^{\mathrm{SVD}}\left(\mathbb{H}, p_{\mathrm{o}}\right)=\max _{\boldsymbol{a} \in \mathcal{R}_{+}^{n_{\mathrm{t}}}} \sum_{i=1}^{n_{\mathrm{t}}} \frac{1}{2} \log \left(1+\frac{2 b_{i, i}^{2} a_{i}^{2}}{\pi e}\right),
$$

where $\boldsymbol{a}$ satisfies $\sum_{i=1}^{n_{\mathrm{t}}} \sum_{j=1}^{n_{\mathrm{t}}}\left|v_{i, j}\right| a_{j} \leq p_{\mathrm{o}}$. Defining $\nu_{j}=$ $\sum_{i=1}^{n_{\mathrm{t}}}\left|v_{i, j}\right|$, one can write this constraint as $\sum_{j=1}^{n_{\mathrm{t}}} a_{j} \nu_{j} \leq p_{\mathrm{o}}$. This problem is similar to the one considered in [33], whose solution is $a_{i} \nu_{i} \in\left\{0, \frac{1}{2 \lambda} \pm \sqrt{\frac{1}{4 \lambda^{2}}-\frac{1}{c_{i}^{2}}}\right\}$, with $c_{i}=\frac{b_{i, i}}{\nu_{i}} \sqrt{\frac{2}{\pi e}}$ and $\lambda>0$ chosen so that $\sum_{i=1}^{n_{\mathrm{t}}} a_{i} \nu_{i}=p_{\mathrm{o}}$. Thus, the optimal $a_{i}$ has the form $a_{i} \in\left\{0, \frac{1}{2 \lambda \nu_{i}} \pm \sqrt{\frac{1}{4 \lambda^{2} \nu_{i}^{2}}-\frac{1}{c_{i}^{2}}}\right\}$.

The optimal solution approaches $a_{i} \nu_{i}=\frac{p_{\mathrm{o}}}{n_{\mathrm{t}}}, \forall i$ as $p_{\mathrm{o}} \rightarrow \infty$. This can be shown similar to [33]. Substituting this in the achievable rate expression, we conclude that

$$
r_{\mathrm{e}}^{\mathrm{SVD}}\left(\mathbb{H}, p_{\mathrm{o}}\right)=\sum_{i=1}^{n_{\mathrm{t}}} \frac{1}{2} \log \left(\frac{2 b_{i, i}^{2} p_{\mathrm{o}}^{2}}{\pi e \nu_{i}^{2} n_{\mathrm{t}}^{2}}\right)+o\left(\log \left(p_{\mathrm{o}}\right)\right) .
$$

This proves Corollary 3 .

\section{CAPACity ANAlysis FOR $n_{\mathrm{t}} \leq n_{\mathrm{r}}$}

\section{A. Capacity Upper Bounds}

1) Proof of Upper Bound $\bar{c}_{1}\left(\mathbb{H}, p_{\mathrm{o}}\right)$ : To derive this bound, we start by multiplying the received signal $\boldsymbol{Y}(k)$ by $\mathbb{Q}$ corresponding to the $\mathrm{QR}$-decomposition $\mathbb{H}=\mathbb{Q R}$. Since $\mathbb{Q}$ is orthogonal, this transformation is invertible, and the transformed channel has the same capacity as the original channel. Thus, the capacity of the channel can be written as $c\left(\mathbb{H}, p_{\mathrm{o}}\right)=\max _{f(\boldsymbol{x})} \mathrm{I}(\boldsymbol{X} ; \tilde{\boldsymbol{Y}})$ where $f(\boldsymbol{x})$ is a distribution of $\boldsymbol{X} \in \mathcal{R}_{+}^{n_{\mathrm{t}}}$ satisfying $\sum_{i=1}^{n_{\mathrm{t}}} \mathrm{E}\left[X_{i}\right] \leq p_{\mathrm{o}}$, and $\tilde{\boldsymbol{Y}}=\mathbb{R} \boldsymbol{X}+\boldsymbol{Z}$ with $\boldsymbol{Z} \sim \mathrm{N}\left(\mathbf{0}_{n_{\mathrm{r}}}, \mathbb{I}_{n_{\mathrm{r}}}\right)$.

Let us write $\tilde{\boldsymbol{Y}}=\left[\tilde{\boldsymbol{Y}}_{1}^{\top}, \tilde{\boldsymbol{Y}}_{2}^{\top}\right]^{\top}$ where $\tilde{\boldsymbol{Y}}_{1} \in \mathcal{R}^{n_{\mathrm{t}}}$ and $\tilde{\boldsymbol{Y}}_{2} \in$ $\mathcal{R}^{n_{\mathrm{r}}-n_{\mathrm{t}}}$. Thus, we can write

$$
\begin{aligned}
\mathrm{I}(\boldsymbol{X} ; \tilde{\boldsymbol{Y}}) & =\mathrm{I}\left(\boldsymbol{X} ; \tilde{\boldsymbol{Y}}_{1}, \tilde{\boldsymbol{Y}}_{2}\right) \\
& =\mathrm{I}\left(\boldsymbol{X} ; \tilde{\boldsymbol{Y}}_{1}\right)+\mathrm{I}\left(\boldsymbol{X} ; \tilde{\boldsymbol{Y}}_{2} \mid \tilde{\boldsymbol{Y}}_{1}\right) \\
& =\mathrm{I}\left(\boldsymbol{X} ; \tilde{\boldsymbol{Y}}_{1}\right),
\end{aligned}
$$

using the chain rule, where the last step follows since $\tilde{\boldsymbol{Y}}_{2}$ is only noise ( $\mathbb{R}$ is upper triangular $n_{\mathrm{r}} \times n_{\mathrm{t}}$ ), which is independent of $\boldsymbol{X}$ and of $\tilde{\boldsymbol{Y}}_{1}$. We can write $\tilde{\boldsymbol{Y}}_{1}=\mathbb{R}_{1} \boldsymbol{X}+\tilde{\boldsymbol{Z}}_{1}$ where $\mathbb{R}_{1}$ is the matrix formed by the first $n_{\mathrm{t}}$ rows of $\mathbb{R}$, and $\tilde{\boldsymbol{Z}}_{1} \sim$ $\mathrm{N}\left(\mathbf{0}_{n_{\mathrm{t}}}, \mathbb{I}_{n_{\mathrm{t}}}\right)$. Since $\mathbb{H}$ is of full column rank $n_{\mathrm{t}}$, then $\mathbb{R}_{1}$ is invertible, and we can write

$$
c\left(\mathbb{H}, p_{\mathrm{o}}\right)=\max _{f(\boldsymbol{x})} \mathrm{I}(\boldsymbol{X} ; \breve{\boldsymbol{Y}}),
$$


where $\breve{\boldsymbol{Y}}=\mathbb{R}_{1}^{-1} \tilde{\boldsymbol{Y}}_{1}=\boldsymbol{X}+\breve{\boldsymbol{Z}}$, and $\breve{\boldsymbol{Z}} \sim \mathrm{N}\left(\mathbf{0}_{n_{\mathrm{t}}}, \mathbb{K}\right)$ where $\mathbb{K}=\mathbb{R}_{1}^{-1} \mathbb{R}_{1}^{-\top}=\mathbb{R}^{-1} \mathbb{R}^{-\top}$. Note that

$$
\mathrm{I}(\boldsymbol{X} ; \breve{\boldsymbol{Y}})=\mathrm{h}(\breve{\boldsymbol{Y}})-\mathrm{h}(\breve{\boldsymbol{Z}}) \leq \sum_{i=1}^{n_{\mathrm{t}}} \mathrm{h}\left(\breve{Y}_{i}\right)-\mathrm{h}(\breve{\boldsymbol{Z}}),
$$

which follows using the chain rule and since conditioning reduces entropy. Let $\bar{Z}_{i}$ be an $\mathrm{N}\left(0, k_{i, i}\right)$ random variable. Adding $\sum_{i=1}^{n_{\mathrm{t}}}\left(\mathrm{h}\left(\breve{Z}_{i}\right)-\mathrm{h}\left(\bar{Z}_{i}\right)\right)=0$ to this upper bound, leads to

$$
\begin{aligned}
\mathrm{I}(\boldsymbol{X} ; \breve{\boldsymbol{Y}}) & \leq \sum_{i=1}^{n_{\mathrm{t}}}\left(\mathrm{h}\left(\breve{Y}_{i}\right)-\mathrm{h}\left(\bar{Z}_{i}\right)\right)+\sum_{i=1}^{n_{\mathrm{t}}} \mathrm{h}\left(\breve{Z}_{i}\right)-\mathrm{h}(\breve{\boldsymbol{Z}}) \\
& =\sum_{i=1}^{n_{\mathrm{t}}} \mathrm{I}\left(X_{i} ; X_{i}+\bar{Z}_{i}\right)+\zeta
\end{aligned}
$$

where $\zeta=\frac{1}{2} \log \left(\frac{\prod_{i=1}^{n_{\mathrm{t}}} k_{i, i}}{|\mathbb{K}|}\right)$. The last step follows since $\bar{Z}_{i}$ and $\breve{Z}_{i}$ have the same distribution and are independent of $X_{i}$. Note that the summation above is the mutual information across a system of parallel channels with input $X_{i}$ and output $X_{i}+\bar{Z}_{i}, i=1, \ldots, n_{\mathrm{t}}$. Consequently,

$$
\begin{aligned}
c\left(\mathbb{H}, p_{\mathrm{o}}\right) & \leq \max _{f(\boldsymbol{x})} \sum_{i=1}^{n_{\mathrm{t}}} \mathrm{I}\left(X_{i} ; X_{i}+\bar{Y}_{i}\right)+\zeta \\
& \leq \max _{\boldsymbol{\xi} \in \mathcal{I}} \sum_{i=1}^{n_{\mathrm{t}}} \max _{f\left(x_{i}\right): \mathrm{E}\left[X_{i}\right] \leq \xi_{i}} \mathrm{I}\left(X_{i} ; X_{i}+\bar{Z}_{i}\right)+\zeta .
\end{aligned}
$$

The inner maximization is the capacity of an averageconstrained IM-DD SISO channel with average intensity $\xi_{i}$ and channel coefficient $k_{i, i}^{-\frac{1}{2}}$. Let this capacity be upper bounded by some function $\bar{r}\left(k_{i, i}^{-\frac{1}{2}}, \xi_{i}\right)$. Thus

$$
c\left(\mathbb{H}, p_{\mathrm{o}}\right) \leq \max _{\boldsymbol{\xi} \in \mathcal{I}} \sum_{i=1}^{n_{\mathrm{t}}} \bar{r}\left(k_{i, i}^{-\frac{1}{2}}, \xi_{i}\right)+\zeta .
$$

Noting that $\mathbb{K}=\mathbb{R}_{1}^{-1} \mathbb{R}_{1}^{-\top}=\left[\mathbb{R}_{1}^{\top} \mathbb{R}_{1}\right]^{-1}=\left[\mathbb{R}^{\top} \mathbb{Q}^{\top} \mathbb{Q} \mathbb{R}\right]^{-1}=$ $\left[\mathbb{H}^{\top} \mathbb{H}\right]^{-1}$ proves the first bound in Theorem 1.

2) Proof of Upper Bound $\bar{c}_{2}\left(\mathbb{H}, p_{\mathrm{o}}\right)$ : We start from $c\left(\mathbb{H}, p_{\mathrm{o}}\right)=\max _{f(\boldsymbol{x})} \mathrm{I}(\boldsymbol{X} ; \boldsymbol{Y})$. We have

$$
\begin{aligned}
\mathrm{I}(\boldsymbol{X} ; \boldsymbol{Y}) & =\mathrm{h}(\boldsymbol{Y})-\mathrm{h}(\boldsymbol{Z}) \\
& \leq \sum_{i=1}^{n_{\mathrm{r}}}\left(\mathrm{h}\left(Y_{i}\right)-\mathrm{h}\left(Z_{i}\right)\right) \\
& =\sum_{i=1}^{n_{\mathrm{r}}}\left(\mathrm{h}\left(W_{i}+Z_{i}\right)-\mathrm{h}\left(Z_{i}\right)\right),
\end{aligned}
$$

where the inequality follows using the chain rule, the independence of $Z_{1}, \ldots, Z_{n_{\mathrm{r}}}$, and the fact that conditioning does not increase entropy, and where $W_{i}=\sum_{j=1}^{n_{\mathrm{t}}} h_{i, j} X_{j}$. Thus,

$$
c\left(\mathbb{H}, p_{\mathrm{o}}\right) \leq \max _{f(\boldsymbol{x})} \sum_{i=1}^{n_{\mathrm{r}}} \mathrm{I}\left(\boldsymbol{X} ; W_{i}+Z_{i}\right) .
$$

Note that $\mathrm{I}\left(\boldsymbol{X} ; W_{i}+Z_{i}\right)$ is the mutual information across a MISO channel with input $\boldsymbol{X}$ and output $W_{i}+Z_{i}$, and the sum above represents a system of parallel MISO channels. But $\mathrm{I}\left(\boldsymbol{X} ; W_{i}+Z_{i}\right) \leq \max _{f\left(w_{i}\right)} \mathrm{I}\left(W_{i} ; W_{i}+Z_{i}\right)$ where the maximization is over all distributions of $W_{i} \geq 0$ (since $h_{i, j} \geq$
$0)$ with $\mathrm{E}\left[W_{i}\right] \leq \hat{\xi}_{i}=\sum_{j=1}^{n_{\mathrm{t}}} h_{i, j} \xi_{j}$. This upper bound is the capacity of an average-constrained IM-DD SISO channel with channel $h=1$ and average constraint $\hat{\xi}_{i}$. Thus, I $\left(\boldsymbol{X} ; W_{i}+\right.$ $\left.Z_{i}\right) \leq \bar{r}\left(1, \hat{\xi}_{i}\right)$ for some SISO capacity upper bound $\bar{r}(\cdot, \cdot)$, and

$$
c\left(\mathbb{H}, p_{\mathrm{o}}\right) \leq \max _{f(\boldsymbol{x})} \sum_{i=1}^{n_{\mathrm{r}}} \bar{r}\left(1, \hat{\xi}_{i}\right)=\max _{\boldsymbol{\xi} \in \mathcal{I}} \sum_{i=1}^{n_{\mathrm{r}}} \bar{r}\left(1, \hat{\xi}_{i}\right),
$$

which proves the second bound in Theorem 1 .

\section{B. High-SNR Capacity}

To characterize the high-SNR capacity of the channel, we first recall from Corollary 2 that $\lim _{p_{\mathrm{o}} \rightarrow \infty}\left(r_{\mathrm{e}}^{\mathrm{QR}}\left(\mathbb{H}, p_{\mathrm{o}}\right)-\frac{1}{2} \log \left|\frac{e p_{\mathrm{o}}^{2}}{2 \pi n_{\mathrm{t}}^{2}} \mathbb{H}^{\mathrm{T}} \mathbb{H}\right|\right)=0$, and hence,

$$
c\left(\mathbb{H}, p_{\mathrm{o}}\right) \geq \frac{1}{2} \log \left|\frac{e p_{\mathrm{o}}^{2}}{2 \pi n_{\mathrm{t}}^{2}} \mathbb{H}^{\top} \mathbb{H}\right|+o\left(\log \left(p_{\mathrm{o}}\right)\right) .
$$

Next, we consider the upper bound in Theorem 1 given by

$$
c\left(\mathbb{H}, p_{\mathrm{o}}\right) \leq \max _{\boldsymbol{\xi} \in \mathcal{I}} \sum_{i=1}^{n_{\mathrm{t}}} \bar{r}\left(k_{i, i}^{-\frac{1}{2}}, \xi_{i}\right)+\zeta,
$$

where $\zeta=\frac{1}{2} \log \left(\frac{\prod_{i=1}^{n_{\mathrm{t}}} k_{i, i}}{|\mathbb{K}|}\right)$ and $\mathbb{K}=\left[\mathbb{H}^{\top} \mathbb{H}\right]^{-1}$. Let us denote the optimal $\boldsymbol{\xi}$ by $\left[\xi_{1, \mathrm{o}}, \ldots, \xi_{n_{\mathrm{t}}, \mathrm{o}}\right]$. We first argue that as $p_{\mathrm{o}} \rightarrow$ $\infty$, we have $\xi_{i, \mathrm{o}} \rightarrow \infty$. A formal proof of this intuition is given in Appendix A. Using this, and the fact that [22]

$$
\bar{r}\left(k_{i, i}^{-\frac{1}{2}}, \xi_{i}\right)=\frac{1}{2} \log \left(\frac{e \xi_{i}^{2}}{2 \pi k_{i, i}}\right)+o\left(\log \left(\xi_{i}\right)\right),
$$

we can write

$$
\begin{aligned}
& \sum_{i=1}^{n_{\mathrm{t}}} \bar{r}\left(k_{i, i}^{-\frac{1}{2}}, \xi_{i, \mathrm{o}}\right) \\
& =\sum_{i=1}^{n_{\mathrm{t}}}\left(\frac{1}{2} \log \left(\frac{e \xi_{i, \mathrm{o}}^{2}}{2 \pi k_{i, i}}\right)+o\left(\log \left(\xi_{i, \mathrm{o}}\right)\right)\right) \\
& =\sum_{i=1}^{n_{\mathrm{t}}} \frac{1}{2} \log \left(\frac{e \xi_{i, \mathrm{o}}^{2}}{2 \pi k_{i, i}}\right)+o\left(\log \left(p_{\mathrm{o}}\right)\right) \\
& \leq \max _{\boldsymbol{\xi} \in \mathcal{I}} \sum_{i=1}^{n_{\mathrm{t}}} \frac{1}{2} \log \left(1+\frac{e \xi_{i}^{2}}{2 \pi k_{i, i}}\right)+o\left(\log \left(p_{\mathrm{o}}\right)\right) .
\end{aligned}
$$

This maximization is not solved by standard water-filling, but can be solved as in [33]. The solution of this maximization converges to $\xi_{i}=\frac{p_{\mathrm{o}}}{n_{\mathrm{t}}}$ as $p_{\mathrm{o}}$ increases [33]. Hence,

$$
\sum_{i=1}^{n_{\mathrm{t}}} \bar{r}\left(k_{i, i}^{-\frac{1}{2}}, \xi_{i, \mathrm{o}}\right) \leq \sum_{i=1}^{n_{\mathrm{t}}} \frac{1}{2} \log \left(\frac{e p_{\mathrm{o}}^{2}}{2 \pi n_{\mathrm{t}}^{2} k_{i, i}}\right)+o\left(\log \left(p_{\mathrm{o}}\right)\right) .
$$

Therefore,

$$
\begin{aligned}
c\left(\mathbb{H}, p_{\mathrm{o}}\right) & \leq \sum_{i=1}^{n_{\mathrm{t}}} \frac{1}{2} \log \left(\frac{e p_{\mathrm{o}}^{2}}{2 \pi n_{\mathrm{t}}^{2} k_{i, i}}\right)+o\left(\log \left(p_{\mathrm{o}}\right)\right)+\zeta \\
& =\frac{1}{2} \log \left|\frac{e p_{\mathrm{o}}^{2}}{2 \pi n_{\mathrm{t}}^{2}} \mathbb{K}^{-1}\right|+o\left(\log \left(p_{\mathrm{o}}\right)\right) \\
& =\frac{1}{2} \log \left|\frac{e p_{\mathrm{o}}^{2}}{2 \pi n_{\mathrm{t}}^{2}} \mathbb{H}^{\top} \mathbb{H}\right|+o\left(\log \left(p_{\mathrm{o}}\right)\right) .
\end{aligned}
$$


Combining (47) and (55), we conclude that

$$
\lim _{p_{\mathrm{o}} \rightarrow \infty}\left(c\left(\mathbb{H}, p_{\mathrm{o}}\right)-\frac{1}{2} \log \left|\frac{e p_{\mathrm{o}}^{2}}{2 \pi n_{\mathrm{t}}^{2}} \mathbb{H}^{\mathrm{T}} \mathbb{H}\right|\right)=0,
$$

which proves Theorem 2.

Remark 4: This asymptotic capacity is achieved using exponentially distributed $X_{i}, i=1, \ldots, n_{\mathrm{t}}$. The geometric distribution which is near-optimal at high SNR for the SISO channel [23] is also near-optimal at high SNR for the MIMO channel in conjunction with the QR scheme.

\section{EXTENSIONS}

The results presented thus far are restricted to averageconstrained IM-DD MIMO channels with $n_{\mathrm{t}} \leq n_{\mathrm{r}}$. In this section, we consider extensions of those results to averageand-peak constrained channels, and channels with $n_{\mathrm{t}}>n_{\mathrm{r}}$. Due to the superiority of the QR scheme discussed above, we restrict our attention to this scheme henceforth.

\section{A. Average and Peak Constraints with $n_{\mathrm{t}} \leq n_{\mathrm{r}}$}

Herein, in addition to the total average intensity constraint, each light aperture is constrained by a peak constraint. For simplicity, we assume that all apertures are identical and thus have the same constraint. Hence, $\boldsymbol{X}$ satisfies $0 \leq X_{i} \leq x_{\max }$ and $\|\boldsymbol{\xi}\|_{1} \leq p_{\mathrm{o}}=\alpha x_{\max }$ for some $\alpha>0$, where $\xi_{i}=\mathrm{E}\left[X_{i}\right] .^{5}$ We call this channel an average-and-peak constrained IM-DD MIMO channel, and denote its capacity by $c_{\text {ap }}\left(\mathbb{H}, p_{\mathrm{o}}, x_{\max }\right)$.

In this case, similar results as for the average-constrained case can be written with minor adjustments. We start with the achievable rate of the $\mathrm{QR}$ scheme.

Definition 2: An average-and-peak constrained IM-DD SISO channel is one with input $x \in\left[0, x_{\max }\right]$ satisfying $\mathrm{E}[X] \leq p_{\mathrm{o}}=\eta x_{\max }$ with $\eta>0$, and output $Y=h x+Z$ where $h \in \mathcal{R}_{+}$and $Z \sim \mathrm{N}(0,1)$. We denote an achievable rate and a capacity upper bound for this channel by $r_{\text {ap }}\left(h, p_{\mathrm{o}}, x_{\max }\right)$ and $\bar{r}_{\mathrm{ap}}\left(h, p_{\mathrm{o}}, x_{\mathrm{max}}\right)$, respectively.

Corollary 4: In an average-and-peak constrained IM-DD MIMO channel with $n_{\mathrm{t}} \leq n_{\mathrm{r}}$, a QR scheme achieves

$$
r_{\mathrm{ap}}^{\mathrm{QR}}\left(\mathbb{H}, p_{\mathrm{o}}, x_{\max }\right)=\max _{\substack{\mathbb{P} \in \mathcal{P} \\ \xi \in \mathcal{I}}} \sum_{i=1}^{n_{\mathrm{t}}} r_{\mathrm{ap}}\left(\left|r_{i, i}^{[\mathbb{P}]}\right|, \xi_{\pi_{i}}, x_{\max }\right),
$$

where $\mathcal{I}, \mathcal{P}, \pi_{i}, r_{i, i}^{[\mathbb{P}]}$ are as defined in Proposition 2, and $r_{\text {ap }}\left(h, \xi, x_{\max }\right)$ is defined in Definition 2.

Proof: This follows by simply replacing $r_{\mathrm{m}}(h, \xi)$ in Proposition 2 with $r_{\text {ap }}\left(h, \xi, x_{\max }\right)$.

Here, we can replace $r_{\text {ap }}\left(h, \xi, x_{\max }\right)$ with any achievable rate over an average-and-peak constrained SISO channel from [22], [24], [41]. The upper bound in Theorem 1 can also be extended to this case as follows.

Corollary 5: The capacity of an average-and-peak constrained IM-DD MIMO channel with a channel matrix $\mathbb{H} \in$ $\mathcal{R}_{+}^{n_{\mathrm{r}} \times n_{\mathrm{t}}}$ with rank $n_{\mathrm{t}} \leq n_{\mathrm{r}}$ is upper bounded by

$$
\bar{c}_{\text {ap } 1}\left(\mathbb{H}, p_{\mathrm{o}}, x_{\max }\right)=\max _{\boldsymbol{\xi} \in \mathcal{I}} \sum_{i=1}^{n_{\mathrm{t}}} \bar{r}_{\mathrm{ap}}\left(k_{i, i}^{-\frac{1}{2}}, \xi_{i}, x_{\max }\right)+\zeta,
$$

\footnotetext{
${ }^{5}$ Due to this, high SNR can be described by $p_{\mathrm{o}} \rightarrow \infty$ or $x_{\max } \rightarrow \infty$.
}

and

$$
\bar{c}_{\mathrm{ap} 2}\left(\mathbb{H}, p_{\mathrm{o}}, x_{\max }\right)=\max _{\boldsymbol{\xi} \in \mathcal{I}} \sum_{i=1}^{n_{\mathrm{r}}} \bar{r}_{\mathrm{ap}}\left(1, \hat{\xi}_{i}, \hat{x}_{\max , i}\right)
$$

where $\zeta, \quad \mathcal{I}, \quad k_{i, i}, \quad \hat{\xi}_{i}$ are as defined in Theorem 1 , $\left[\hat{x}_{\max , 1}, \ldots, \hat{x}_{\max , n_{\mathrm{r}}}\right]^{\top}=\mathbb{H}\left[x_{\max }, \ldots, x_{\max }\right]^{\top}$, and $\bar{r}_{\mathrm{ap}}(\cdot, \cdot, \cdot)$ is defined in Definition 2.

Proof: This follows similar to Theorem 1 with $\bar{r}(h, \xi)$ replaced with $\bar{r}_{\text {ap }}\left(h, \xi, x_{\text {max }}\right)$.

Examples of $\bar{r}_{\text {ap }}\left(h, \xi, x_{\max }\right)$ can be found in [22], [24]. In what follows, we use

$$
\bar{r}_{\mathrm{ap}}\left(h, \xi, x_{\max }\right)=\min \left\{\bar{r}(h, \xi), \bar{r}_{\mathrm{p}}\left(h, x_{\max }\right)\right\},
$$

where $\bar{r}(h, \xi)$ is the average-constrained SISO channel capacity upper bound defined in Theorem 1 , and $\bar{r}_{\text {ap }}\left(h, x_{\max }\right)$ is a peak-constrained SISO channel capacity upper bound given by [24]

$$
\begin{aligned}
& \bar{r}_{\mathrm{p}}\left(h, x_{\max }\right) \\
& =\sup _{\delta \in[0,1]}\left(\frac{\delta}{2} \log \left(\frac{h^{2} x_{\max }^{2}}{2 \pi e}\right)-\log \left((1-\delta)^{\frac{3(1-\delta)}{2}} \delta^{\delta}\right)\right) .
\end{aligned}
$$

Next, we compare the bounds considering two cases: $p_{\mathrm{o}} \geq$ $n_{\mathrm{t}} \frac{x_{\max }}{2}$ and $p_{\mathrm{o}}<n_{\mathrm{t}} \frac{x_{\max }}{2}$, i.e., $\alpha \geq \frac{n_{\mathrm{t}}}{2}$ and $\alpha<\frac{n_{\mathrm{t}}}{2}$, respectively.

1) Case $\alpha \geq \frac{n_{t}}{2}$ : In this case, the average constraint becomes redundant, and it is optimal to replace $\xi_{i}$ by $\frac{x_{\max }}{2}$ $\forall i \in\left\{1, \ldots, n_{\mathrm{t}}\right\}$. This follows from [24, Lemma 1]. Thus, we can restrict our attention to this solution, and the maximization with respect to $\boldsymbol{\xi}$ in (57) can be dropped. At this point, we have a set of parallel channels with channel coefficients $\left|r_{1,1}^{[\mathbb{P}]}\right|, \ldots,\left|r_{n_{\mathrm{t}}, n_{\mathrm{t}}}^{[\mathbb{P}}\right|$, where the input over each subchannel satisfies $0 \leq X_{i} \leq x_{\max }$ and $\mathrm{E}\left[X_{i}\right]=\frac{x_{\max }}{2}$. This allows us to replace $r_{\mathrm{ap}}\left(\left|r_{i, i}^{[\mathbb{P}]}\right|, \frac{x_{\max }}{2}, x_{\max }\right)$ in (57) with $r_{\mathrm{m}}\left(\left|r_{i, i}^{[\mathbb{P}]}\right|, \frac{x_{\max }}{2}\right), \mathrm{m} \in\{\mathrm{u}, \mathrm{p}\}$, defined in Lemma 2. Thus, we have the achievable rate

$$
r_{\mathrm{ap}, \mathrm{m}}^{\mathrm{QR}}\left(\mathbb{H}, p_{\mathrm{o}}, x_{\mathrm{max}}\right)=\max _{\mathbb{P} \in \mathcal{P}} \sum_{i=1}^{n_{\mathrm{t}}} r_{\mathrm{m}}\left(\left|r_{i, i}^{[\mathbb{P}]}\right|, \frac{x_{\max }}{2}\right),
$$

for $m \in\{u, p\}$.

Using a continuous uniform input distribution, i.e., $\mathrm{m}=\mathrm{u}$, leads to a more tractable expression, where we can show that maximization in (62) with respect to $\mathbb{P}$ is not needed at high SNR. Namely, we have the following statement as a parallel to Corollary 2.

Corollary 6: The achievable rate $r_{\mathrm{ap}, \mathrm{u}}^{\mathrm{QR}}\left(\mathbb{H}, p_{\mathrm{o}}, x_{\max }\right)$ satisfies the following independent of $\mathbb{P}$ :

$$
\lim _{x_{\max } \rightarrow \infty}\left(r_{\mathrm{ap}, \mathrm{u}}^{\mathrm{QR}}\left(\mathbb{H}, p_{\mathrm{o}}, x_{\max }\right)-\frac{1}{2} \log \left|\frac{x_{\max }^{2}}{2 \pi e} \mathbb{H}^{\top} \mathbb{H}\right|\right)=0 .
$$


Proof: Starting with (62), we have

$$
\begin{aligned}
& r_{\mathrm{ap}, \mathrm{u}}^{\mathrm{QR}}\left(\mathbb{H}, p_{\mathrm{o}}, x_{\max }\right) \\
& =\max _{\mathbb{P} \in \mathcal{P}} \sum_{i=1}^{n_{\mathrm{t}}} \frac{1}{2} \log \left(1+\frac{\left(r_{i, i}^{[\mathbb{P}]} x_{\max }\right)^{2}}{2 \pi e}\right) \\
& =\max _{\mathbb{P} \in \mathcal{P}} \sum_{i=1}^{n_{\mathrm{t}}} \frac{1}{2} \log \left(\frac{\left(r_{i, i}^{[\mathbb{P}]} x_{\max }\right)^{2}}{2 \pi e}\right)+o\left(\log \left(x_{\max }\right)\right) \\
& =\max _{\mathbb{P} \in \mathcal{P}} \frac{1}{2} \log \left(\frac{x_{\max }^{2 n_{\mathrm{t}}}}{(2 \pi e)^{n_{\mathrm{t}}}} \prod_{i=1}^{n_{\mathrm{t}}}\left(r_{i, i}^{[\mathbb{P}]}\right)^{2}\right)+o\left(\log \left(x_{\max }\right)\right) \\
& =\frac{1}{2} \log \left|\frac{x_{\max }^{2}}{2 \pi e} \mathbb{H}^{\top} \mathbb{H}\right|+o\left(\log \left(x_{\max }\right)\right),
\end{aligned}
$$

independent of $\mathbb{P}$. This proves the desired result.

This asymptotic achievable rate coincides with capacity at high SNR as stated next.

Theorem 3: For an average-and-peak constrained IM-DD MIMO channel with a full column rank $\mathbb{H} \in \mathcal{R}_{+}^{n_{\times} \times n_{\mathrm{t}}}$ and with $\alpha \geq \frac{n_{t}}{2}$, the capacity satisfies

$$
\lim _{x_{\max } \rightarrow \infty}\left(c_{\text {ap }}\left(\mathbb{H}, p_{\mathrm{o}}, x_{\max }\right)-\frac{1}{2} \log \left|\frac{x_{\max }^{2}}{2 \pi e} \mathbb{H}^{\top} \mathbb{H}\right|\right)=0,
$$

and is asymptotically achievable using the QR scheme.

Proof: We have $\bar{r}_{\text {ap }}\left(h, \xi, x_{\max }\right) \leq \bar{r}_{\mathrm{p}}\left(h, x_{\max }\right)$, which in turn converges to $\frac{1}{2} \log \left(\frac{h^{2} x_{\max }^{2}}{2 \pi e}\right)$ as $x_{\max } \rightarrow \infty$. Thus, we can write $\bar{r}_{\text {ap }}\left(h, \xi, x_{\max }\right) \leq \frac{1}{2} \log \left(\frac{h^{2} x_{\max }^{2}}{2 \pi e}\right)+o\left(\log \left(x_{\max }\right)\right)$ leading to

$$
\begin{aligned}
& \bar{c}_{\mathrm{ap} 1}\left(\mathbb{H}, p_{\mathrm{o}}, x_{\max }\right) \\
& \leq \sum_{i=1}^{n_{\mathrm{t}}} \frac{1}{2} \log \left(\frac{x_{\max }^{2}}{2 \pi e k_{i, i}}\right)+\frac{1}{2} \log \left(\frac{\prod_{i=1}^{n_{\mathrm{t}}} k_{i, i}}{|\mathbb{K}|}\right)+o\left(\log \left(x_{\max }\right)\right) \\
& =\sum_{i=1}^{n_{\mathrm{t}}} \frac{1}{2} \log \left|\frac{x_{\max }^{2}}{2 \pi e} \mathbb{H}^{\top} \mathbb{H}\right|+o\left(\log \left(x_{\max }\right)\right) .
\end{aligned}
$$

Since the achievable rate $r_{\mathrm{ap}, \mathrm{u}}^{\mathrm{QR}}\left(\mathbb{H}, x_{\max }\right)$ has the same asymptotic expression (Corollary 6), it coincides with the upper bound at high SNR, which proves the statement.

Remark 5: Theorem 3 also provides the high-SNR capacity for a MIMO channel with peak constraints only, which is captured by the case $p_{\mathrm{o}}=n_{\mathrm{t}} x_{\max }$.

Fig. 3a shows the capacity upper bounds in Corollary 5 in addition to the achievable rate $r_{\mathrm{ap}, \mathrm{m}}^{\mathrm{QR}}\left(\boldsymbol{H}, x_{\max }\right)$ in (62), wherein the required optimizations are done numerically. It shows how upper bound $1\left(\bar{c}_{\mathrm{ap} 1}\left(\mathbb{H}, p_{\mathrm{o}}, x_{\max }\right)\right)$ coincides with the achievable rates at high SNR, verifying Theorem 3. Upper bound $2\left(\bar{c}_{\mathrm{ap} 2}\left(\mathbb{H}, p_{\mathrm{o}}, x_{\max }\right)\right)$ is tighter than upper bound 1 at lower SNR.

2) Case $\alpha<\frac{n_{t}}{2}$ : In this case, the average constraint is active for at least one component of $\boldsymbol{X}$. We can replace $r_{\text {ap }}(\cdot, \cdot, \cdot)$ in (57) with the rate achievable using a truncated-exponential [22], truncated geometric [41], or truncated-Gaussian distribution [24]. Instead of repeating their achievable rate expressions, we recall the following simple observation from [24].
Lemma 3: For an average-and-peak constrained IM-DD SISO channel as defined in Definition 2, the achievable rate using a truncated-Gaussian distributed $X$ satisfies

$$
\begin{aligned}
& r_{\mathrm{tg}}\left(h, p_{\mathrm{o}}, x_{\max }\right) \\
& =\frac{1}{2} \log \left(1+\frac{h^{2}}{2 \pi e} \min \left\{e^{2} p_{\mathrm{o}}^{2}, x_{\max }^{2}\right\}\right)-0.1+o\left(\log \left(x_{\max }\right)\right) .
\end{aligned}
$$

Using this lemma, we can write the achievable rate

$$
r_{\mathrm{ap}, \mathrm{tg}}^{\mathrm{QR}}\left(\mathbb{H}, p_{\mathrm{o}}, x_{\max }\right)=\max _{\substack{\mathbb{P} \in \mathcal{P} \\ \boldsymbol{\xi} \in \mathcal{I}}} \sum_{i=1}^{n_{\mathrm{t}}} r_{\mathrm{tg}}\left(\left|r_{i, i}^{[\mathbb{P}]}\right|, \xi_{\pi_{i}}, x_{\max }\right),
$$

which leads to the following statements.

Corollary 7: The achievable rate $r_{\mathrm{ap}, \mathrm{tg}}^{\mathrm{QR}}\left(\mathbb{H}, p_{\mathrm{o}}, x_{\max }\right)$ satisfies the following independent of $\mathbb{P}$ :

$$
\begin{aligned}
\lim _{x_{\max } \rightarrow \infty} & \left(r_{\mathrm{ap}, \mathrm{tg}}^{\mathrm{QR}}\left(\mathbb{H}, p_{\mathrm{o}}, x_{\max }\right)\right. \\
& \left.-\frac{1}{2} \log \left|\frac{e}{2 \pi} \min \left\{\frac{p_{\mathrm{o}}^{2}}{n_{\mathrm{t}}^{2}}, \frac{x_{\max }^{2}}{e^{2}}\right\} \mathbb{H}^{\top} \mathbb{H}\right|+0.1 n_{\mathrm{t}}\right)=0 .
\end{aligned}
$$

Proof: The achievable rate in (69) can be rewritten as

$$
\begin{aligned}
& r_{\mathrm{ap}, \mathrm{tg}}^{\mathrm{QR}}\left(\mathbb{H}, p_{\mathrm{o}}, x_{\max }\right) \\
& =\max _{\substack{\mathbb{P} \in \mathcal{P} \\
\boldsymbol{\xi} \in \mathcal{J}}} \sum_{i=1}^{n_{\mathrm{t}}} \frac{1}{2}\left(1+\frac{e\left(r_{i, i}^{[\mathbb{P}]} \xi_{\pi_{i}}\right)^{2}}{2 \pi}\right)+0.1 n_{\mathrm{t}}+o\left(\log \left(x_{\max }\right)\right),
\end{aligned}
$$

where $\mathcal{J}=\mathcal{I} \cap\left[0, \frac{x_{\max }}{e}\right]^{n_{\mathrm{t}}}$. The optimal solution of (71) with respect to $\boldsymbol{\xi}$ approaches $\xi_{i}=\min \left\{\frac{p_{\mathrm{o}}}{n_{\mathrm{t}}}, \frac{x_{\max }}{e}\right\}$ as $x_{\max }$ increases (cf. [33, Sec. VI.B.2]). Thus,

$$
\begin{aligned}
& r_{\mathrm{ap} 2}^{\mathrm{QR}}\left(\mathbb{H}, p_{\mathrm{o}}, x_{\max }\right) \\
&=\max _{\mathbb{P} \in \mathcal{P}} \sum_{i=1}^{n_{\mathrm{t}}} \frac{1}{2} \log \left(\frac{e\left(r_{i, i}^{[\mathbb{P}]}\right)^{2}}{2 \pi} \min \left\{\frac{p_{\mathrm{o}}^{2}}{n_{\mathrm{t}}^{2}}, \frac{x_{\max }^{2}}{e^{2}}\right\}\right) \\
&-0.1 n_{\mathrm{t}}+o\left(\log \left(x_{\max }\right)\right) \\
&= \max _{\mathbb{P} \in \mathcal{P}} \frac{1}{2} \log \left(\left(\frac{e}{2 \pi} \min \left\{\frac{p_{\mathrm{o}}^{2}}{n_{\mathrm{t}}^{2}}, \frac{x_{\max }^{2}}{e^{2}}\right\}\right)^{n_{\mathrm{t}}} \prod_{i=1}^{n_{\mathrm{t}}}\left(r_{i, i}^{[\mathbb{P}]}\right)^{2}\right) \\
&-0.1 n_{\mathrm{t}}+o\left(\log \left(x_{\max }\right)\right) \\
&= \frac{1}{2} \log \left|\frac{e}{2 \pi} \min \left\{\frac{p_{\mathrm{o}}^{2}}{n_{\mathrm{t}}^{2}}, \frac{x_{\max }^{2}}{e^{2}}\right\} \mathbb{H}^{\top} \mathbb{H}\right|-0.1 n_{\mathrm{t}} \\
&+o\left(\log \left(x_{\max }\right)\right),
\end{aligned}
$$

independent of $\mathbb{P}$. This proves the desired result.

Theorem 4: For an average-and-peak constrained IM-DD MIMO channel with a full column rank $\mathbb{H} \in \mathcal{R}_{+}^{n_{\times} \times n_{\mathrm{t}}}$ and with $\alpha<\frac{n_{t}}{2}$, the capacity satisfies

$$
\begin{aligned}
\lim _{x_{\max } \rightarrow \infty}\left(c_{\mathrm{ap}}\left(\mathbb{H}, p_{\mathrm{o}}, x_{\max }\right)\right. & \\
- & \left.\frac{1}{2} \log \left|\frac{e}{2 \pi} \min \left\{\frac{p_{\mathrm{o}}^{2}}{n_{\mathrm{t}}^{2}}, \frac{x_{\max }^{2}}{e^{2}}\right\} \mathbb{H}^{\top} \mathbb{H}\right|\right) \leq 0.1 n_{\mathrm{t}} .
\end{aligned}
$$

Proof: Suppose that $p_{\mathrm{o}} \leq \frac{n_{\mathrm{t}} x_{\max }}{e}$. In this case, we have $\bar{c}_{\mathrm{ap} 1}\left(\mathbb{H}, p_{\mathrm{o}}\right) \leq \bar{c}_{1}\left(\mathbb{H}, p_{\mathrm{o}}\right)$ defined in Theorem 1. This upper bound and the asymptotic achievable rate in Corollary 7 are at most $0.1 n_{\mathrm{t}}$ nats apart at high SNR. If $p_{\mathrm{o}}>\frac{n_{\mathrm{t}} x_{\max }}{e}$, we have that $\bar{c}_{\text {ap } 1}\left(\mathbb{H}, p_{\mathrm{o}}, x_{\max }\right)$ is upper bounded by (67). By 


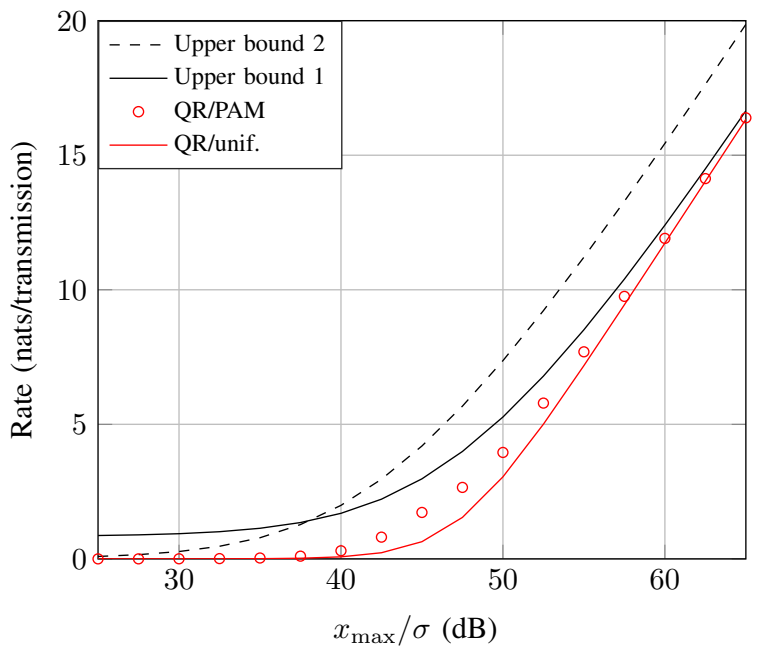

(a) $\alpha=\frac{n_{\mathrm{t}}}{2}$.

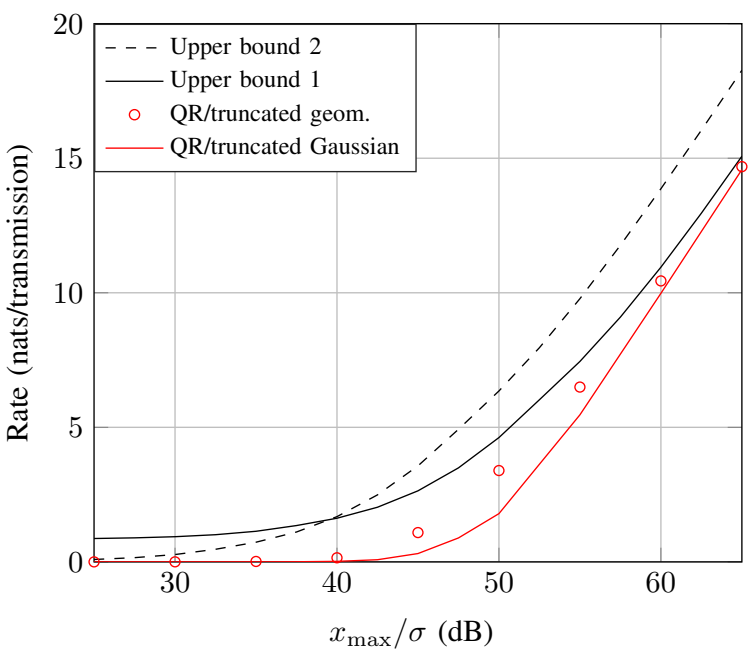

(b) $\alpha=\frac{n_{\mathrm{t}}}{4}$.

Fig. 3: Achievable rates and upper bounds for the MIMO channel described in Table II as Case a.

comparing this asymptotic upper bound with the asymptotic achievable rate in Corollary 7, we can see that the two are at most $0.1 n_{\mathrm{t}}$ nats apart. This proves the statement.

Fig. 3b shows the capacity upper bounds in Corollary 5, in addition to the achievable rate $r_{\mathrm{ap}}^{\mathrm{QR}}\left(\mathbb{H}, p_{\mathrm{o}}, x_{\max }\right)$ given in Corollary 7 with $r_{\text {ap }}(\cdot, \cdot, \cdot)$ replaced with the SISO achievable rate using a truncated-Gaussian input distribution [24] and a truncated-geometric input distribution [41]. The intensity allocation in the achievable rates is obtained using a grid search. Another possibility is to use the algorithm in [33] which uses a surrogate function to obtain a simple and reliable allocation. The figure shows how the upper bound $\bar{c}_{\mathrm{ap} 1}$ and the achievable rates are within a small gap at high $\operatorname{SNR}\left(<0.1 n_{\mathrm{t}}\right)$, thus verifying Theorem 4 . Next, we discuss how the results can be extended to $n_{\mathrm{t}}>n_{\mathrm{r}}$.

\section{B. MIMO channel with $n_{\mathrm{t}}>n_{\mathrm{r}}$}

The advantage of the case $n_{\mathrm{t}} \leq n_{\mathrm{r}}$ is that the pseudoinverse of $\mathbb{H}$ exists. This is useful for describing the channelinversion receiver, and also for deriving capacity bounds. This is unfortunately not the case if $n_{\mathrm{t}}>n_{\mathrm{r}}$, which we discuss in this subsection.

1) Achievable Rate: The QR scheme can be applied here if we convert the channel to an effective $n_{\mathrm{r}} \times n_{\mathrm{r}}$ channel. This can be done e.g. by deactivating $n_{\mathrm{t}}-n_{\mathrm{r}}$ transmit apertures or repeating some signals over multiple apertures. These and other possibilities and others can be described by a general positive precoding. Consider an average-constrained channel, and let the vector intended for transmission in time $k$ be $\boldsymbol{s}(k) \in \mathcal{R}_{+}^{n_{\mathrm{r}}}$ whose $i^{\text {th }}$ component $s_{i}(k)$ is a realization of a random variable $S_{i} \geq 0$ with $\mathrm{E}\left[S_{i}\right]=\xi_{i}$ and $\sum_{i=1}^{n_{\mathrm{r}}} \xi_{i} \leq p_{\mathrm{o}}$. This is precoded into $\boldsymbol{x}(k)=\mathbb{G} \boldsymbol{s}(k)$, where $\mathbb{G} \in \mathcal{R}_{+}^{n_{+} \times n_{\mathrm{r}}}$ satisfies

$$
\operatorname{rank}(\mathbb{G})=n_{\mathrm{r}}, \text { and } \sum_{i=1}^{n_{\mathrm{t}}} g_{i, j} \leq 1 \text { for } j \in\left\{1, \ldots, n_{\mathrm{r}}\right\} .
$$

This precoding preserves both nonnegativity and sum intensity, and leads to a received signal $\boldsymbol{Y}(k)=\mathbb{H} \mathbb{G} \boldsymbol{s}(k)+\boldsymbol{Z}(k)$. Since $\mathbb{H} G \in \mathcal{R}_{+}^{n_{r} \times n_{\mathrm{r}}}$ has rank $n_{\mathrm{r}}$, the QR scheme can be applied.

Under both average and peak constraints, we choose $S_{i} \in$ $\left[0, x_{\max }\right]$ for $i \in\left\{1, \ldots, n_{\mathrm{r}}\right\}$ with $\mathrm{E}\left[S_{i}\right]=\xi_{i}$ and $\sum_{i=1}^{n_{\mathrm{r}}} \xi_{i} \leq$ $p_{\mathrm{o}}$. Then, the matrix $\mathbb{G}$ has to satisfy the additional constraint

$$
\sum_{j=1}^{n_{\mathrm{r}}} g_{i, j} \leq 1 \text { for } i \in\left\{1, \ldots, n_{\mathrm{r}}\right\}
$$

so that $\boldsymbol{x}(k)$ also satisfies the peak constraint $x_{i}(k) \leq x_{\max }$. This leads to the following statement.

Proposition 4: In an average-constrained IM-DD MIMO channel with $n_{\mathrm{t}}>n_{\mathrm{r}}$, a QR scheme achieves

$$
\tilde{r}_{\mathrm{m}}^{\mathrm{QR}}\left(\mathbb{H}, p_{\mathrm{o}}\right)=\max _{\mathbb{G} \in \mathcal{G}} r_{\mathrm{m}}^{\mathrm{QR}}\left(\mathbb{H} \mathbb{G}, p_{\mathrm{o}}\right), \quad \mathrm{m} \in\{\mathrm{e}, \mathrm{g}\},
$$

where $r_{\mathrm{m}}^{\mathrm{QR}}(\cdot, \cdot)$ is defined in Proposition 2, and $\mathcal{G}=\{\mathbb{G} \in$ $\mathcal{R}_{+}^{n_{\mathrm{t}} \times n_{\mathrm{r}}} \mid(77)$ is satisfied $\}$. Under average and peak constraints, a QR scheme achieves

$$
\tilde{r}_{\mathrm{ap}}^{\mathrm{QR}}\left(\mathbb{H}, p_{\mathrm{o}}, x_{\max }\right)=\max _{\mathbb{G} \in \mathcal{G}_{\mathrm{ap}}} r_{\text {ap }}^{\mathrm{QR}}\left(\mathbb{H} \mathbb{G}, p_{\mathrm{o}}, x_{\max }\right),
$$

where $r_{\mathrm{ap}}^{\mathrm{QR}}(\cdot, \cdot, \cdot)$ is defined in Corollary 4 , and $\mathcal{G}_{\text {ap }}=\{\mathbb{G} \in$ $\mathcal{R}_{+}^{n_{\mathrm{t}} \times n_{\mathrm{r}}} \mid(77) \&(78)$ are satisfied $\}$.

Proof: This follows from Proposition 2 and Corollary 4 with additional optimization with respect to $\mathbb{G}$.

This scheme is not precoding-free anymore due to $\mathbb{G}$. Note that $\mathbb{G}$ takes care of the decoding order, and hence, a permutation matrix similar to $\mathbb{P}$ in Proposition 2 is not needed.

The high-SNR asymptote of $\tilde{r}_{\mathrm{m}}^{\mathrm{QR}}\left(\mathbb{H}, p_{\mathrm{o}}\right)$ can be obtained by replacing $\mathbb{H}$ in Corollary 2 by $\mathbb{H} \mathbb{G}$. The resulting high-SNR achievable rate is the solution of

$$
\max _{\mathbb{G} \in \mathcal{G}} \frac{1}{2} \log \left|\frac{e p_{\mathrm{o}}^{2}}{2 \pi n_{\mathrm{r}}^{2}} \mathbb{G}^{\top} \mathbb{H}^{\top} \mathbb{H} \mathbb{G}\right| .
$$

This is an interesting problem for future research. A similar optimization arises for $\tilde{r}_{\mathrm{ap}}^{\mathrm{QR}}\left(\mathbb{H}, p_{\mathrm{o}}, x_{\mathrm{max}}\right.$ ) (average and peak constraints) with $\frac{e p_{\mathrm{o}}^{2}}{2 \pi n_{\mathrm{r}}^{2}}$ in (81) replaced with $\frac{x_{\max }^{2}}{2 \pi e}$ if $p_{\mathrm{o}} \geq$ 
$\frac{n_{\mathrm{r}} x_{\max }}{2}$. Otherwise, this is replaced with $\frac{e}{2 \pi} \min \left\{\frac{p_{\mathrm{o}}^{2}}{n_{\mathrm{r}}^{2}}, \frac{x_{\max }^{2}}{e^{2}}\right\}$, and $0.1 n_{\mathrm{r}}$ is subtracted from the achievable rate as in Corollary 7.

2) Upper Bound: The upper bounds $\bar{c}_{1}\left(\mathbb{H}, p_{\mathrm{o}}\right)$ and $\bar{c}_{\text {ap } 1}\left(\mathbb{H}, p_{\mathrm{o}}, x_{\max }\right)$ given in Theorems 1 and 5 , respectively, do not hold in this case since $n_{\mathrm{r}}<n_{\mathrm{t}}$. However, the bounds $\bar{c}_{2}\left(\mathbb{H}, p_{\mathrm{o}}\right)$ and $\bar{c}_{\mathrm{ap} 2}\left(\mathbb{H}, p_{\mathrm{o}}, x_{\max }\right)$ continue to hold. Thus, we have the following.

Theorem 5: The capacity of an average-constrained IM-DD MIMO channel with $\mathbb{H} \in \mathcal{R}_{+}^{n_{\mathrm{r}} \times n_{\mathrm{r}}}$ with rank $n_{\mathrm{r}}<n_{\mathrm{t}}$ is upper bounded by $\bar{c}_{2}\left(\mathbb{H}, p_{\mathrm{o}}\right)$ (Theorem 1$)$. Under peak and average constraints, capacity is upper bounded by $\bar{c}_{\mathrm{ap} 2}\left(\mathbb{H}, p_{\mathrm{o}}, x_{\max }\right)$ (Theorem 5).

3) Comparison: Fig. 4 shows the bounds versus SNR for a $4 \times 2$ MIMO channel with $\mathbb{H}$ given by the first two rows of $\mathbb{H}_{\mathrm{a}}$ in Table III. The achievable rate in Fig. 4a is obtained from Proposition 4 with an exponential input distribution $(\mathrm{m}=\mathrm{e})$, with numerical maximization with respect to $\mathbb{G}$ (gridsearch), and using the JA intensity allocation algorithm in [33]. The achievable rate in Fig. $4 \mathrm{~b}$ is obtained using a truncated Gaussian input distribution, with numerical maximization with respect to $\mathbb{G}$ and $\boldsymbol{\xi}$ (grid-search). The upper bounds are from Theorem 5.

Based on Propositions 4 and Theorem 5, we obtain the following statement.

Theorem 6: For an IM-DD MIMO channel with a full row rank $\mathbb{H} \in \mathcal{R}_{+}^{n_{\mathrm{r}} \times n_{\mathrm{t}}}$, the capacity satisfies $c\left(\mathbb{H}, p_{\mathrm{o}}\right)=n_{\mathrm{r}} \log \left(p_{\mathrm{o}}\right)+o\left(\log \left(p_{\mathrm{o}}\right)\right)$ under an average constraint, $c_{\text {ap }}\left(\mathbb{H}, p_{\mathrm{o}}, x_{\max }\right)=n_{\mathrm{r}} \log \left(x_{\max }\right)+o\left(\log \left(x_{\max }\right)\right)$ under both average and peak constraints.

Proof: From (81), we have that $c\left(\mathbb{H}, p_{\mathrm{o}}\right) \geq$ $\frac{1}{2} \log \left|\frac{e p_{\mathrm{o}}^{2}}{2 \pi n_{\mathrm{r}}^{2}} \mathbb{G}^{\top} \mathbb{H}^{\top} \mathbb{H} \mathbb{G}\right|+o\left(\log \left(p_{\mathrm{o}}\right)\right)$ for some $\mathbb{G}$ so that $\mathbb{H G}$ has rank $n_{\mathrm{r}}$. On the other hand, from Theorem 5, we have the upper bound $\bar{c}_{2}\left(\mathbb{H}, p_{\mathrm{o}}\right)=\max _{\boldsymbol{\xi} \in \mathcal{I}} \sum_{i=1}^{n_{\mathrm{r}}} \bar{r}\left(1, \hat{\xi}_{i}\right)$ where $\left[\hat{\xi}_{1}, \ldots, \hat{\xi}_{n_{\mathrm{r}}}\right]^{\top}=\mathbb{H}\left[\xi_{1}, \ldots, \xi_{n_{\mathrm{t}}}\right]^{T}$. Comparing the lower bound above and this upper bound, and using similar arguments as in Appendix A, we can show that the optimal $\hat{\xi}_{i}$ denoted $\hat{\xi}_{i, \mathrm{o}}$ satisfies $\hat{\xi}_{i, \mathrm{o}} \rightarrow \infty$ as $p_{\mathrm{o}} \rightarrow \infty \forall i$. Then, using (49), we can write $c\left(\mathbb{H}, p_{\mathrm{o}}\right) \leq \sum_{i=1}^{n_{\mathrm{r}}} \frac{1}{2} \log \left(\frac{e \hat{\xi}_{i, \mathrm{o}}^{2}}{2 \pi}\right)+o\left(\log \left(p_{\mathrm{o}}\right)\right)$. But $\hat{\xi}_{i, \mathrm{o}} \leq p_{\mathrm{o}}\|\boldsymbol{h}\|_{1}$ where $\boldsymbol{h}_{i}$ is the $i^{\text {th }}$ row of $\mathbb{H}$. Thus $c\left(\mathbb{H}, p_{\mathrm{O}}\right) \leq \sum_{i=1}^{n_{\mathrm{r}}} \frac{1}{2} \log \left(\frac{e p_{\mathrm{o}}^{2}\left\|\boldsymbol{h}_{i}\right\|_{1}^{2}}{2 \pi}\right)+o\left(\log \left(p_{\mathrm{o}}\right)\right)$ which concludes the proof. The result under both average and peak constraints follows similarly using (68) and (60).

Finally, the following high-SNR capacity for the MISO case follows from Proposition 4 and Theorem 5. We denote $\mathbb{H}$ by $\boldsymbol{h}^{\top}=\left[h_{1}, \ldots, h_{n_{\mathrm{t}}}\right]$ since in this case $\mathbb{H}$ is a $1 \times n_{\mathrm{t}}$ vector.

Corollary 8: For an IM-DD MISO channel $\left(n_{\mathrm{t}}>n_{\mathrm{r}}=1\right)$ under an average constraint only, the capacity satisfies

$$
\lim _{p_{\mathrm{o}} \rightarrow \infty}\left(c\left(\boldsymbol{h}^{\top}, p_{\mathrm{o}}\right)-\frac{1}{2} \log \left(\frac{e p_{\mathrm{O}}^{2} \max _{i} h_{i}^{2}}{2 \pi}\right)\right)=0 .
$$

Proof: We have the achievable rate $\max _{\boldsymbol{g}} r_{\mathrm{e}}^{\mathrm{QR}}\left(\boldsymbol{h}^{\top} \boldsymbol{g}, p_{\mathrm{o}}\right)$ from Proposition 4 , where $\boldsymbol{g} \in \mathcal{R}_{+}^{n_{\mathrm{t}} \times 1}$ with $\|\boldsymbol{g}\|_{1} \leq 1$. Since $\boldsymbol{h}^{\top} \boldsymbol{g}$ is a scalar, then,

$$
\begin{aligned}
\max _{\boldsymbol{g}} \frac{1}{2} \log \left(\frac{e p_{\mathrm{O}}^{2}\left(\boldsymbol{h}^{\top} \boldsymbol{g}\right)^{2}}{2 \pi}\right) & =\frac{1}{2} \log \left(\frac{e p_{\mathrm{O}}^{2}\left(\max _{\boldsymbol{g}} \boldsymbol{h}^{\top} \boldsymbol{g}\right)^{2}}{2 \pi}\right) \\
& =\frac{1}{2} \log \left(\frac{e p_{\mathrm{O}}^{2} \max _{i} h_{i}^{2}}{2 \pi}\right) \cdot(83)
\end{aligned}
$$

On the other hand, from Theorem 5, we have the upper bound $\bar{c}_{2}\left(\boldsymbol{h}^{\top}, p_{\mathrm{o}}\right)=\max _{\boldsymbol{\xi} \in \mathcal{I}} \bar{r}\left(1, \boldsymbol{h}^{\top} \boldsymbol{\xi}\right)$. By [22], we have $\bar{r}\left(1, \boldsymbol{h}^{\top} \boldsymbol{\xi}\right)=\frac{1}{2} \log \left(\frac{e\left(\boldsymbol{h}^{T} \boldsymbol{\xi}\right)^{2}}{2 \pi}\right)+o\left(\log \left(\boldsymbol{h}^{T} \boldsymbol{\xi}\right)\right)$. Simple manipulations lead to

$$
\begin{aligned}
\bar{c}_{2}\left(\boldsymbol{h}^{\top}, p_{\mathrm{o}}\right) & =\frac{1}{2} \log \left(\frac{e\left(\max _{\boldsymbol{\xi} \in \mathcal{I}} \boldsymbol{h}^{\top} \boldsymbol{\xi}\right)^{2}}{2 \pi}\right)+o\left(\log \left(p_{\mathrm{o}}\right)\right) \\
& =\frac{1}{2} \log \left(\frac{e p_{\mathrm{O}}^{2} \max _{i} h_{i}^{2}}{2 \pi}\right)+o\left(\log \left(p_{\mathrm{o}}\right)\right)
\end{aligned}
$$

which coincides with the achievable rate at high SNR and concludes the proof.

\section{CONCLUSION}

We studied several MIMO schemes for IM-DD systems in terms of their achievable rates. The SVD-based precoding scheme - which is optimal in RF MIMO - must be modified to a DC-offset SVD-based scheme in IM-DD MIMO, leading to sub-optimal performance. To avoid this deterioration, precoding-free schemes are favored in IM-DD MIMO. We have derived achievable rates of channel inversion and QRdecomposition based schemes. We have shown that the QRbased scheme has superior performance, and is optimal at high SNR if the transmitters has no more apertures than the receiver. Thereby, we characterized the channel's high-SNR capacity for this case. The result holds under an average or peak intensity constraint. We have also extended the results to the case with both average and peak intensity constraints, where we characterized the high-SNR capacity within a small gap. We also extended the capacity bounds to the case with more transmit than receive apertures. In this case, the achievable rates and upper bounds do not coincide, thus highlighting an interesting problem for further future investigation.

\section{APPENDIX A}

The Optimal $\xi_{i}$ IN (48) GOES TO Infinity $\forall i$ AS $p_{\mathrm{o}} \rightarrow \infty$

Capacity is upper bounded by

$$
\bar{c}_{1}\left(\mathbb{H}, p_{\mathrm{o}}\right)=\max _{\boldsymbol{\xi} \in \mathcal{I}} \sum_{i=1}^{n_{\mathrm{t}}} \bar{r}\left(k_{i, i}^{-\frac{1}{2}}, \xi_{i}\right)+\frac{1}{2} \log \left(\frac{\prod_{i=1}^{n_{\mathrm{t}}} k_{i, i}}{|\mathbb{K}|}\right),
$$

by Theorem 1. Let the optimal solution of the maximization above be denoted by $\left[\xi_{1, \mathrm{o}}, \ldots, \xi_{n_{\mathrm{t}}, \mathrm{o}}\right]$. We follow a proof by contradiction, in which we assume that $\xi_{j, o}$ is bounded for some $j$ as $p_{\mathrm{o}} \rightarrow \infty$, i.e., $\xi_{j, \mathrm{o}} \leq a_{j}$ from some constant $a_{j}$, while the remaining $\xi_{i, \mathrm{o}}, i \neq j$ are not. Then we show that this assumption is false. We have

$$
\bar{r}\left(k_{j, j}^{-\frac{1}{2}}, \xi_{j, \mathrm{o}}\right) \leq b\left(k_{j, j}^{-\frac{1}{2}} \xi_{j, \mathrm{o}}, 1,0\right) \leq b\left(k_{j, j}^{-\frac{1}{2}} a_{j}, 1,0\right) \triangleq b_{j},
$$




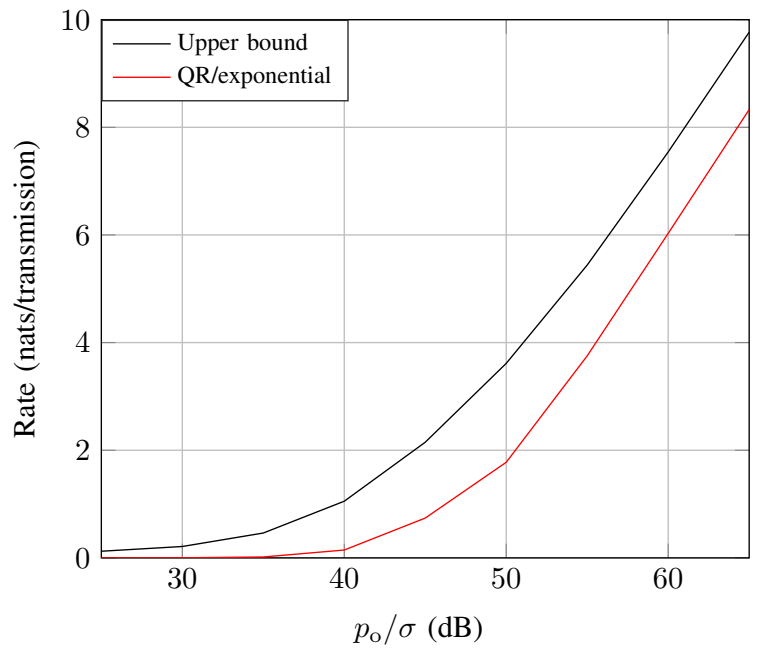

(a) Average constraint only.

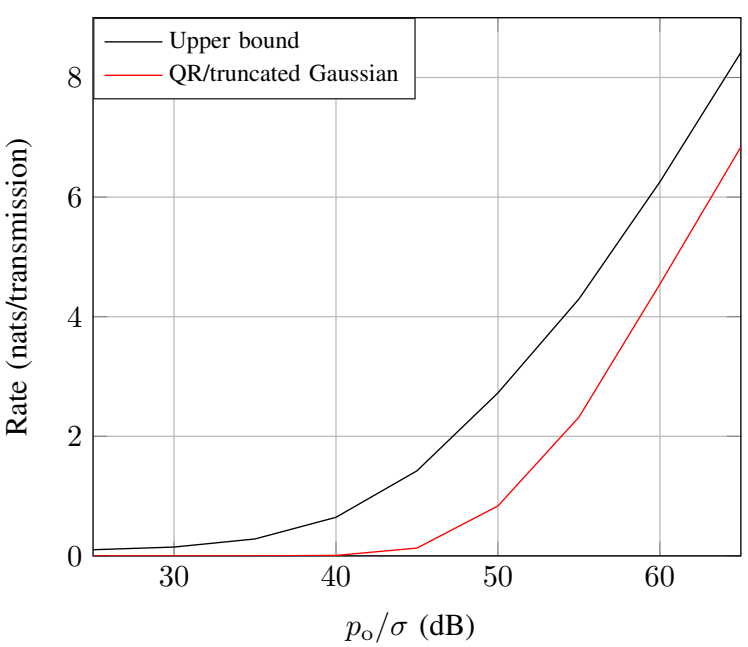

(b) Average and peak constraints: $\alpha=\frac{n_{\mathrm{r}}}{4}$.

Fig. 4: Achievable rates and capacity upper bounds for a $4 \times 2$ MIMO channel with $\mathbb{H}$ given by the first two rows of $\mathbb{H}_{\mathrm{a}}$ in Table III.

(see (18)). Thus,

$$
\begin{aligned}
& \max _{\boldsymbol{\xi} \in \mathcal{I}} \sum_{i=1}^{n_{\mathrm{t}}} \bar{r}\left(k_{i, i}^{-\frac{1}{2}}, \xi_{i}\right) \\
& \leq \sum_{i=1, i \neq j}^{n_{\mathrm{t}}} \bar{r}\left(k_{i, i}^{-\frac{1}{2}}, \xi_{i, \mathrm{o}}\right)+b_{j} \\
& =\sum_{i=1, i \neq j}^{n_{\mathrm{t}}}\left(\frac{1}{2} \log \left(\frac{e \xi_{i, \mathrm{o}}^{2}}{2 \pi k_{i, i}}\right)+o\left(\log \left(\xi_{i, \mathrm{o}}\right)\right)\right)+b_{j} \\
& =\sum_{i=1, i \neq j}^{n_{\mathrm{t}}} \frac{1}{2} \log \left(\frac{e \xi_{i, \mathrm{o}}^{2}}{2 \pi k_{i, i}}\right)+b_{j}+o\left(\log \left(p_{\mathrm{o}}\right)\right),
\end{aligned}
$$

using (49). Next, note that $\sum_{i=1, i \neq j}^{n_{\mathrm{t}}} \xi_{i, \mathrm{o}} \leq p_{\mathrm{o}}$. Then, subject to this constraint, we can write

$$
\begin{aligned}
& \max _{\boldsymbol{\xi} \in \mathcal{I}} \sum_{i=1}^{n_{\mathrm{t}}} \bar{r}\left(k_{i, i}^{-\frac{1}{2}}, \xi_{i}\right) \\
& \leq \max _{\xi_{i}: i \neq j} \sum_{i=1, i \neq j}^{n_{\mathrm{t}}} \frac{1}{2} \log \left(\frac{e \xi_{i}^{2}}{2 \pi k_{i, i}}\right)+b_{j}+o\left(\log \left(p_{\mathrm{o}}\right)\right) \\
& =\sum_{i=1, i \neq j}^{n_{\mathrm{t}}} \frac{1}{2} \log \left(\frac{e p_{\mathrm{o}}^{2}}{2 \pi\left(n_{\mathrm{t}}-1\right)^{2} k_{i, i}}\right)+b_{j}+o\left(\log \left(p_{\mathrm{o}}\right)\right),
\end{aligned}
$$

where we used [33] which shows that the optimal solution of this maximization converges to equal allocation as $p_{\mathrm{o}}$ increases. Substituting in the upper bound, and after some manipulations, we obtain

$$
\begin{aligned}
& \bar{c}_{1}\left(\mathbb{H}, p_{\mathrm{o}}\right) \\
& \leq \frac{n_{\mathrm{t}}-1}{2} \log \left(\frac{e p_{\mathrm{o}}^{2}}{2 \pi n_{\mathrm{t}}^{2}}\right)+\frac{1}{2} \log \left|\mathbb{H}^{\top} \mathbb{H}\right|+\psi+o\left(\log \left(p_{\mathrm{o}}\right)\right),
\end{aligned}
$$

where $\psi=\left(n_{\mathrm{t}}-1\right) \log \left(\frac{n_{\mathrm{t}}}{n_{\mathrm{t}}-1}\right)+b_{j}+\frac{1}{2} \log \left(k_{j, j}\right)$. But the following rate is achievable using the $\mathrm{QR}$-decomposition scheme (47)

$$
c\left(\mathbb{H}, p_{\mathrm{o}}\right) \geq \frac{n_{\mathrm{t}}}{2} \log \left(\frac{e p_{\mathrm{o}}^{2}}{2 \pi n_{\mathrm{t}}^{2}}\right)+\frac{1}{2} \log \left|\mathbb{H}^{\top} \mathbb{H}\right| .
$$

This lower bound eventually becomes larger than the upper bound (89) as $p_{\mathrm{o}}$ increases, leading to a contradiction. Thus, the assumption that $\xi_{j, \mathrm{o}}$ is bounded is false. The same holds if more than one $\xi_{i, \mathrm{o}}$ is bounded, which concludes the proof.

\section{APPENDIX B}

\section{GAP TO HIGH-SNR CAPACITY OF CI AND DC-SVD}

The gap-to-optimality of the CI scheme can be expressed at high SNR by comparing Theorem 2 and Corollary 1. This leads to a gap $\delta^{\mathrm{CI}} \stackrel{p_{\mathrm{o}} \rightarrow \infty}{\longrightarrow} \frac{1}{2} \log \left(\left|\mathbb{H}^{\mathrm{T}} \mathbb{H}\right| \prod_{i=1}^{n_{\mathrm{t}}}\left\|\boldsymbol{t}_{i}\right\|^{2}\right)$. Thus, the CI scheme requires $\frac{10}{n_{\mathrm{t}}} \log _{10}\left(\left|\mathbb{H}^{\top} \mathbb{H}\right|^{\frac{1}{2}} \prod_{i=1}^{n_{\mathrm{t}}}\left\|\boldsymbol{t}_{i}\right\|\right) \mathrm{dB}$ extra $\frac{p_{\mathrm{o}}}{\sigma}$ to achieve the same rate as the $\mathrm{QR}$ scheme at high SNR. This calculation is confirmed by Fig. 2. Note that this high-SNR gap is always positive since $\prod_{i=1}^{n_{\mathrm{t}}}\left\|\boldsymbol{t}_{i}\right\|^{2} \geq$ $\left|\mathbb{T T}^{\top}\right|=\left|\left(\mathbb{H}^{\top} \mathbb{H}\right)^{-1}\right|=\left|\mathbb{H}^{\top} \mathbb{H}\right|^{-1}$ by Hadamard's inequality, with equality if and only if $\mathbb{H}^{\top} \mathbb{H}$ is diagonal, which holds if and only if $\mathbb{H}$ has orthogonal columns. Since $h_{i, j} \geq 0, \mathbb{H}$ has orthogonal columns if and only if it has one nonzero value in each row, for which the MIMO channel reduces to a system of parallel channels [33]. Thus, CI is only optimal if the MIMO channel has no cross-talk.

Similarly, the gap-to-optimality of the DC-SVD scheme satisfies $\delta^{\mathrm{SVD}} \stackrel{p_{\mathrm{o}} \rightarrow \infty}{\longrightarrow} \frac{1}{2} \log \left(\prod_{i=1}^{n_{\mathrm{t}}} \frac{e^{2} \nu_{i}^{2}}{4}\right)$, which is obtained by comparing Theorem 2 and Corollary 3, where $\nu_{i}$ is defined below (14), and where we used the fact that $\prod_{i=1}^{n_{\mathrm{t}}} k_{i, i}^{-1}=\left|\mathbb{H}^{\top} \mathbb{H}\right|=\prod_{i=1}^{n_{\mathrm{t}}} b_{i, i}^{2}$. This gap is equivalent to $\frac{10}{n_{\mathrm{t}}} \log _{10}\left(\prod_{i=1}^{n_{\mathrm{t}}} \frac{e \nu_{i}}{2}\right) \mathrm{dB}$ extra $\frac{p_{\mathrm{o}}}{\sigma}$, which is confirmed by Fig. 2. Similar to $\delta^{\mathrm{CI}}$, this high-SNR gap is the smallest when $\mathbb{H}$ corresponds to parallel channels. To see this, note that $\nu_{i}^{2}=\left(\sum_{j=1}^{n_{\mathrm{t}}}\left|v_{j, i}\right|\right)^{2} \geq \sum_{j=1}^{n_{\mathrm{t}}} v_{j, i}^{2}=1$ since $\mathbb{V}$ is an orthogonal matrix. Therefore, $\prod_{i=1}^{n_{\mathrm{t}}} \frac{e^{2} \nu_{i}^{2}}{4} \geq \frac{e^{2 n_{\mathrm{t}}}}{4^{n_{\mathrm{t}}}}$ with equality 
when $\mathbb{V}=\mathbb{I}_{n_{\mathrm{t}}}$. But this implies that $\mathbb{H}=\mathbb{U B}$ and hence, it has orthogonal columns. Since $\mathbb{H}$ has positive components, this can only be the case if $\mathbb{H}$ has a parallel-channel structure, i.e., it has one nonzero component in each row.

\section{REFERENCES}

[1] A. Chaaban, Z. Rezki, and M.-S. Alouini, "MIMO intensity-modulation channels: Capacity bounds and high SNR characterization," in Proc. IEEE Int. Conf. Commun. (ICC), Paris, France, May 2017, pp. 1-6.

[2] M. A. Khalighi and M. Uysal, "Survey on free space optical communications: A communication theory perspective," Commun. Surveys Tuts., vol. 16, no. 4, pp. 2231-2258, 4th quarter 2014.

[3] H. Burchardt, N. Serafimovski, D. Tsonev, S. Videv, and H. Haas, "VLC: Beyond point-to-point communication," IEEE Commun. Mag., vol. 52, no. 7, pp. 98-105, July 2014.

[4] D. Karunatilaka, F. Zafar, V. Kalavally, and R. Parthiban, "LED based indoor visible light communications: State of the art," IEEE Commun. Surveys Tuts., vol. 17, no. 3, pp. 1649-1678, 3rd quarter 2015.

[5] P. Pathak, X. Fen, P. Hu, and P. Mohapatra, "Visible light communication, networking, and sensing: A survey, potential and challenges," IEEE Commun. Surveys Tuts., vol. 17, no. 4, pp. 2047-2077, 4th quarter 2015.

[6] D. K. Borah, A. C. Boucouvalas, C. C. Davis, S. Hranilovic, and K. Yiannopoulos, "A review of communication-oriented optical wireless systems," EURASIP J. Wireless Commun. Netw., vol. 2012, no. 1, pp. $1-28,2012$.

[7] F. Demers, H. Yanikomeroglu, and M. St-Hilaire, "A survey of opportunities for free space optics in next generation cellular networks," in Proc. 9th Annu. Commun. Netw. Services Research Conf. (CNSR), May 2011, pp. 210-216.

[8] M. Khalighi, N. Schwartz, N. Aitamer, and S. Bourennane, "Fading reduction by aperture averaging and spatial diversity in optical wireless systems," IEEE J. Opt. Commun. Netw., vol. 1, no. 6, pp. 580-593, November 2009.

[9] N. Letzepis and A. G. i Fàbregas, "Outage probability of the Gaussian MIMO free-space optical channel with PPM," IEEE Trans. Commun. vol. 57, no. 12, pp. 3682-3690, Dec. 2009.

[10] M. Safari, MIMO Free-Space Optical Communication. Springer International Publishing, 2016.

[11] D. Tse and P. Viswanath, Fundamentals of Wireless Communications. Cambridge University Press, 2005.

[12] D. Bushuev and S. Arnon, "Analysis of the performance of a wireless optical multi-input to multi-output communication system," J. Opt. Soc. Am. A, vol. 23, no. 7, pp. 1722-1730, July 2006.

[13] G. Yang, M.-A. Khalighi, T. Virieux, S. Bourennane, and Z. Ghassemlooy, "Contrasting space-time schemes for MIMO FSO systems with non-coherent modulation," in Proc. Int. Workshop Opt. Wireless Commun. (IWOW), Pisa, Italy, Oct. 2012, pp. 1-3.

[14] E. Başar, E. Panayirci, M. Uysal, and H. Haas, "Generalized LED index modulation optical OFDM for MIMO visible light communications systems," in Proc. of IEEE Int. Conf. Commun. (ICC), May 2016, pp. $1-5$.

[15] K. Xu, H. Y. Yu, Y. J. Zhu, and H. B. Cai, "Channel-adaptive spacecollaborative constellation design for MIMO VLC with fast maximum likelihood detection," IEEE Access, vol. 5, pp. 842-852, Mar. 2017.

[16] H. Marshoud, D. Dawoud, V. M. Kapinas, G. K. Karagiannidis, S. Muhaidat, and B. Sharif, "MU-MIMO precoding for VLC with imperfect CSI," in Proc. Int. Workshop Opt. Wireless Commun. (IWOW), Sep. 2015, pp. 93-97.

[17] L. Zeng, D. O'brien, H. Minh, G. Faulkner, K. Lee, D. Jung, Y. Oh, and E. T. Won, "High data rate multiple input multiple output (MIMO) optical wireless communications using white LED lighting," IEEE $J$. Sel. Areas Commun., vol. 27, no. 9, pp. 1654-1662, Dec. 2009.

[18] T. Fath and H. Haas, "Performance comparison of MIMO techniques for optical wireless communications in indoor environments," IEEE Trans. Commun., vol. 61, no. 2, pp. 733-742, Feb. 2013.

[19] Y. Sapenov, A. Chaaban, Z. Rezki, and M.-S. Alouini, "On the Optimality of Repetition Coding among Rate-1 DC-offset STBCs for MIMO Optical Wireless Communications," KAUST Technical Report http://hdl.handle.net/10754/624894, June 2017.

[20] S. M. Haas and J. H. Shapiro, "Capacity of the multiple-input, multipleoutput Poisson channel," in Stochastic Theory and Control, ser. Lecture Notes in Control and Information Sciences, B. Pasik-Duncan, Ed Springer Berlin Heidelberg, 2002, vol. 280, pp. 155-168.

[21] _ "Capacity of wireless optical communications," IEEE J. Sel. Areas Commun., vol. 21, no. 8, pp. 1346-1357, Oct. 2003.
[22] A. Lapidoth, S. M. Moser, and M. Wigger, "On the capacity of freespace optical intensity channels," IEEE Trans. Inf. Theory, vol. 55, no. 10 , pp. 4449-4461, Oct. 2009.

[23] A. A. Farid and S. Hranilovic, "Capacity bounds for wireless optical intensity channels with Gaussian noise," IEEE Trans. Inf. Theory, vol. 56, no. 12 , pp. 6066-6077, Dec. 2010.

[24] A. Chaaban, J.-M. Morvan, and M.-S. Alouini, "Free-space optical communications: Capacity bounds, approximations, and a new spherepacking perspective," IEEE Trans. Commun., vol. 64, no. 3, pp. 11761191, Mar. 2016

[25] J. M. Kahn and J. R. Barry, "Wireless infrared communications," Proc. of the IEEE, vol. 85, no. 2, pp. 265-298, Feb. 1997.

[26] E. Monteiro and S. Hranilovic, "Design and implementation of colorshift keying for visible light communications," J. Lightw. Technol., vol. 32, no. 10, pp. 2053-2060, May 2014.

[27] S. M. Moser, M. Mylonakis, L. Wang, and M. Wigger, "Asymptotic capacity results for MIMO wireless optical communication," in Proc. IEEE Int. Symp. Inf. Theory (ISIT), Aachen, Germany, Jun. 2017, pp. $536-540$.

[28] S. M. Moser, "Capacity results of an optical intensity channel with inputdependent Gaussian noise," IEEE Trans. Inf. Theory, vol. 58, no. 1, pp. 207-223, Jan. 2012

[29] P. Butala, H. Elgala, and T. Little, "SVD-VLC: A novel capacity maximizing VLC MIMO system architecture under illumination constraints,' in Proc. IEEE Globecom Workshops, Atlanta GA, USA, Dec. 2013, pp. 1087-1092.

[30] K. H. Park, Y. C. Ko, and M. S. Alouini, "On the power and offset allocation for rate adaptation of spatial multiplexing in optical wireless MIMO channels," IEEE Trans. Commun., vol. 61, no. 4, pp. 1535-1543, Apr. 2013.

[31] M. Arar and A. Yongacoglu, "Efficient detection algorithm for $2 \mathrm{Nx} 2 \mathrm{~N}$ MIMO systems using alamouti code and QR decomposition," IEEE Commun. Lett., vol. 10, no. 12, pp. 819-821, Dec. 2006.

[32] H. Kazemi and H. Haas, "Downlink cooperation with fractional frequency reuse in DCO-OFDMA optical attocell networks," in Proc. IEEE Int. Conf. Commun. (ICC), Kuala Lumpur, Malaysia, May 2016, pp. 1-6.

[33] A. Chaaban, Z. Rezki, and M.-S. Alouini, "Fundamental limits of parallel optical wireless channels: Capacity results and outage formulation,' IEEE Trans. Commun., vol. 65, no. 1, pp. 296-311, Jan. 2017.

[34] _ - "Capacity bounds for parallel optical wireless channels," in Proc. of IEEE Int. Conf. Commun. (ICC), Kuala Lumpur, Malaysia, May 2016, pp. $1-6$.

[35] S. Haas, "Capacity of and coding for multiple-aperture, wireless, optical communications," Ph.D. dissertation, Massachusetts Institute of Technology, Cambridge, Massachusetts, USA, 2003.

[36] S. M. Moser, "Duality-based bounds on channel capacity," Ph.D. dissertation, Swiss Fedral Institute of Technology, Zürich, Switzerland, 2005.

[37] S. M. Moser, L. Wang, and M. Wigger, "Asymptotic high-SNR capacity of MISO optical intensity channels," in Proc. IEEE Inf. Theory Workshop (ITW), Kaohsiung, Taiwan, Nov. 2017, pp. 6-10.

[38] A. A. Farid and S. Hranilovic, "Outage capacity optimization for freespace optical links with pointing errors," IEEE/OSA J. Lightw. Technol., vol. 25 , no. 7, pp. 1702-1710, Jul. 2007.

[39] K. H. Park, W. G. Alheadary, and M. S. Alouini, "A novel mirror diversity receiver for indoor MIMO visible light communication systems," in Proc. of IEEE 27th Ann. Int. Symp. Personal, Indoor, and Mobile Radio Commun. (PIMRC), Valencia, Spain, Sep. 2016, pp. 1-6.

[40] T. Cover and J. Thomas, Elements of Information Theory (Second Edition). John Wiley and Sons, Inc., 2006.

[41] A. A. Farid and S. Hranilovic, "Channel capacity and non-uniform signalling for free-space optical intensity channels," IEEE J. Sel. Areas Commun., vol. 27, no. 9, pp. 1-12, Dec. 2009. 


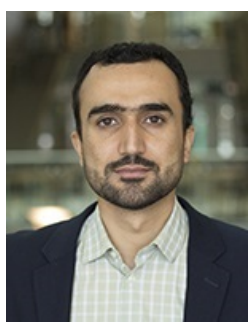

Anas Chaaban (S'09 - M'14 - SM'17) received the Maîtrise ès Sciences degree in electronics from Lebanese University, Lebanon, in 2006, the M.Sc. degree in communications technology and the Dr. Ing. (Ph.D.) degree in electrical engineering and information technology from the University of Ulm and the Ruhr-University of Bochum, Germany, in 2009 and 2013, respectively. From 2008 to 2009, he was with the Daimler AG Research Group On Machine Vision, Ulm, Germany. He was a Research Assistant with the Emmy-Noether Research Group on Wireless Networks, University of Ulm, Germany, from 2009 to 2011, which relocated to the Ruhr-University of Bochum in 2011. He was a PostDoctoral Researcher with the Ruhr-University of Bochum from 2013 to 2014, and with King Abdullah University of Science and Technology from 2015 to 2017. He joined the School of Engineering at the University of British Columbia as an Assistant Professor in 2018. His research interests are in the areas of information theory and wireless communications.

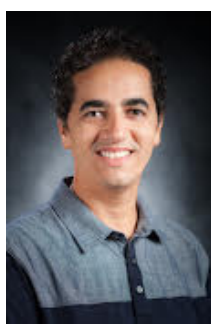

Zouheir Rezki (S'01-M'08-SM'13) was born in Casablanca, Morocco. He received the Diplôme d'Ingénieur degree from the École Nationale de l'Industrie Minérale (ENIM), Rabat, Morocco, in 1994, the M.Eng. degree from École de Technologie Supérieure, Montreal, Québec, Canada, in 2003, and the $\mathrm{Ph} . \mathrm{D}$. degree in electrical engineering from Ecole Polytechnique, Montreal, Québec, in 2008. After a few years of experience as a postdoctoral fellow and a research scientist at KAUST, he joined University of Idaho as an Assistant Professor in the ECE

Department.

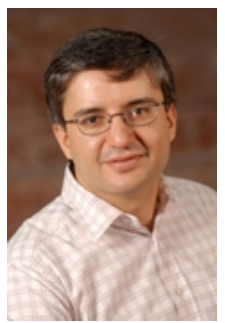

Mohamed-Slim Alouini (S'94, M'98, SM'03, F09) was born in Tunis, Tunisia. He received the Ph.D. degree in Electrical Engineering from the California Institute of Technology (Caltech), Pasadena, CA, USA, in 1998. He served as a faculty member in the University of Minnesota, Minneapolis, MN, USA, then in the Texas A\&M University at Qatar, Education City, Doha, Qatar before joining King Abdullah University of Science and Technology (KAUST), Thuwal, Makkah Province, Saudi Arabia as a Professor of Electrical Engineering in 2009. His current research interests include the modeling, design, and performance analysis of wireless communication systems. 